\title{
Education as a Heart Failure Intervention: What ProvidersTaught Patients in One Hospital Setting
}

\author{
${ }^{1}$ Dr. Theresa Galakatos, ${ }^{2}$ Dr. Michael Bleich, ${ }^{3}$ Dr. Gretchen Drinkard, \\ ${ }^{4}$ Dr. Michael Ward, ${ }^{5}$ Dr. Jean Davis, ${ }^{6}$ Dr. Michael Vaughn \\ ${ }^{1}$ Assistant Professor of Nursing \\ ${ }^{2}$ President, Dean, and Professor of Nursing \\ ${ }^{3}$ Senior Associate Dean and Professor of Nursing \\ ${ }^{4}$ Vice Dean and Professor of Nursing \\ ${ }^{5}$ Senior Associate Dean, Director, and Professor of Nursing \\ ${ }^{6}$ Director and Professor of Social Work
}

\begin{abstract}
This study examined what heart failure $(H F)$ information was provided to patients by health care providers (HCPs), what instructional content taught went beyond the American Heart Association (AHA) Guidelines, and what HF education expert providers viewed as critical for their patients to receive. There is compelling evidence for utilizing educational instruction that includes evidence based guidelines from the AHA.No studieswere found that compared and contrasted these AHA Guidelines with the instructional content provided to hospitalized patients. Using naturalistic inquiry, $10 \mathrm{HF}$ patients (New York Heart Association functional class of III or IV) and 161 HCPs were observed. Results showed that not all AHA topics were consistently covered; $70 \%(n=10)$ patients received less than one hour of instruction, compared to the AHA standard of 60 minutes;AHA Guidelines did not include instructional content on current plan of care which nearly equaled the time spent on AHA topics; brochures and handouts were rarely used and the topics within these materials were not inclusive of AHA standards; asequence of education provided on medications, symptom recognition, diet, and follow up, was congruent with AHA Guidelines and reinforced by the expert panel; and physicians and nurses delivered the most instruction (AHA or non-AHA). The findings of a study of this nature are not generalizable, but still important for future research in testing HF education in other settings for a more robust picture of the benefits of structured versus just in time instruction.
\end{abstract}

Keywords: heart failure, educational interventions, coordination, guidelines

\section{INTRODUCTION}

Heart failure (HF) affects approximately 5.7 million people in the United States (US) and by 2030, projections show a $25 \%$ increase in prevalence (Go et al., 2012, 2013). For patients with HF, there are few solutions to this highly morbid, disabling, and costly disease. To reduce the risk of complications and disease progression, patients hospitalized with HF require education prior to discharge. Education must focus on goals of treatment, prevention of clinical deterioration, and management of ongoing care needs.

Heart failure, a clinical syndrome, is the inability of the heart to pump effectively at rest or during exercise and may profoundly impact activities of daily living and quality of life (Grady et al., 2000). People with HF are a population where lifestyle (poor diet, lack of exercise, smoking) or genetic predisposition (heart valve disease, ventricular dysfunction, high cholesterol) place them at risk for symptom exacerbation, rapid decompensation, frequenthospitalizations, surgeries and procedures, a shortened length of life, or sudden death.The daily reality for people living with HFis a sense of perpetual clinical vulnerability and includes symptoms such asvisible swelling of the legs, face, and abdomen; shortness of breath; breathing difficulty with activity; fatigue; nausea; cough and lung congestion; and weight gain due to fluid retention (Krumholz et al., 2000). Slowing down or preventing target organ damage, or even reversing disease progression requires early evaluation, testing, ongoing treatment, and clinical management.

A strategy that can decrease clinical deterioration or acute instability is a functional partnership between the HF patient and the health care team. Patients and those who support the patient in HF management (i.e. medication administration, meal preparation, symptom monitoring) should be included in a collaborative partnership with the HF team. AHF team mayconsist of two or more of the following: physicians (primary care, cardiology, or otherspecialists), nurse and/or nurse practitioners, mental health professionals, case manager or social workers, dieticians, physical and occupational therapists, pharmacists, and others (American Heart Association [AHA], 2012). Each team member can explore and address a range ofcare needs and respond to urgent concerns tied to HF.

Unlike other disease conditions, HF signs and symptoms are critical indicators of the heart's current capacity to adapt to lifestyle/daily activities making it critical totreat changing symptoms as they arise. 
Reportingchanging symptoms to a healthcare provider islifesaving and results in fewer hospital visits for crisis management (Desai, 2012; Fallis, Dhalla, Klemensberg, \& Bell, 2013). Patient education, therefore, is critical and must include an awareness of self, methods to adapt to life activities based on the daily presentation of the disease state, knowing which symptoms can be self-managed and which symptoms require a report to providers of care. Not surprisingly, the American Heart Association (AHA) evidence-based guidelines support enhanced HF patient education in the hospital prior to discharge (AHA, 2011; Baker et al., 2011).

Early detection and treatment of $\mathrm{HF}$ and its symptoms along with adaptive clinical strategies to manage the symptoms, can reduce acute distress on the diseased organ and improve the patient's quality of life. Patient education is an important clinical intervention from the onset through the duration of $\mathrm{HF}$.

As noted, HF is a progressive syndrome. The New York Heart Association (NYHA) has created a model that classifies the disease from stage $1-4$. This classification schema aids clinicians and patients in understanding the various aims of treatment tied to each stage. The NYHA clinical treatment and patient education guidelines reflect an evolving disease process and guide clinicians through a staged idealized pharmacotherapeutic and non-pharmacotherapeutic clinical treatment plan. The person with HFprogressively advances from one stage to the next unless treatment slows or stops the progression of the disease. As part of HF education, the AHA recommends lifestyle modificationeducation for hospitalized HF patients(AHA, 2011). These AHA guidelines offera framework for healthcare providers (HCPs) to engage with the patient on education that reviews prevention, treatment, and disease progression.To highlight HF progression and demonstrate the role that patient education plays in various disease stages, three reality-based hypothetical cases are presented.

In the first case, a 65-year-old maleis ranked asaNYHA Class II, which indicatesHF withmild symptoms and slight physical limitations. The patient entered the hospital for an uncomplicated inguinal hernia surgery. It was notedthat his HF status was stable on admissionas well as at the time of discharge. On hospital discharge, the patient was told to stop Lasix and drink more fluids to accommodate his lowered blood pressure; what followed on the first night home was difficulty breathing whenlying flat. He adapted to the symptoms by sleeping in a recliner to ease his difficulty breathing. By morning,a four-pound weight gain was registered and swelling in his feet was noted.Knowing these symptoms were a sign of a heart in failure, his provider was called, medications were adjusted, and vigilant monitoring between the patient and provider initiated. The culprit: fluid overload.

The second case is a70-year-old male ranked as a NYHA Class IV reflecting severe symptoms and discomfort when carrying out physical activities. At this stage, the patient has worked with his HF team to engage in palliative care, an approach to clinical management that intensively monitors and manages pain (Goodlin, 2009). The patient is home bound, on a continuous 2 liters of oxygen per nasal cannula, andtakesthe maximum doses of prescribedheartmedications. Even at rest, the patient is short of breath with a look of fear in his eyes. He is worried about what is happening to him. His wifeis part of the caregiving team and, with education, knowsto call the HCP. The result: increased dosing of diuretic medicines to 60 milligrams twice a day, increased oxygen to 4 liters, a planned home visit by the palliative care nurse for evaluation, oxygen saturation assessment,and blood work.The outcome of the activated and adjusted care plan led to lessened patient and caregiver anxiety, improved patient comfort, and appreciated emotional and spiritual support for both the patient and his wife.

The final caseillustrates symptom severityand how an ongoing partnership between aHF patient and the HF healthcare team is necessary. This case involves an adult female who failed to engage in necessary follow-up care from a congenital heart problem corrected by surgery and now is in HF (Reardon \&Pillutia, 2013). At age two, the patient had a Fontan procedure performed to treat a heart defect where blood from the right atrium was surgically directed to the pulmonary arteries to avoid stressing an atrophic, non-functioning right ventricle.Aftersurgery, good heart function was exhibited andthe pediatric surgeons, her parents, and the health care team provided close monitoring.At age 17 shereceived an ablation for an arrhythmia which led the patient to developing a sense of feeling invincible as she left for college before working fora large airline. At age 24 the patient developed shortness of breath, ignoring her body and HF symptoms. After 3 weeks, she went to the emergency room for a cold. In the ER, an arrhythmia (atrial flutter) was discovered and a transesophageal echocardiogram performed. During this procedure a cardiac arrestoccurred, CPR was initiated, a pulse wasregained within a minute, and the patient was cardioverted out of the arrhythmia. After inpatient monitoring, the patient was discharged with a clinical plan which, because of her overall clinical symptoms, necessitated plans for a heart transplant. This demonstrates the role of education, follow-up care, strong connection needed between a patient and the health care team over the span of time, the importance of ongoing HCP-patient communication, self-awareness tied to symptomology, and a willingness to act based on an understanding of what is at stake. 


\section{Incidence and Prevalence}

As previously noted, approximately 5.7 million people have HF in the US (Go et al., 2012). National data from the Centers for Disease Control and Prevention (CDC), which commingles HF with heart disease (a more encompassing category that includes HF, coronary artery disease, arrhythmia, congenital heart defects, peripheral vascular disease, and valvular disease), reveals approximately 600,000 deaths every year in the United States related to heart disease (with HFbeing the primary cause in 55,000 deaths and a contributing cause in 280,000 deaths; Rogers et al., 2012; Kochanek, Xu, Murphy, Miniño, \& Kung, 2011). The Missouri Department of Health and Senior Services (MDHSS) reported heart disease as the leading cause of death statewide with more than 15,000 deaths per year. Locally, heart disease is a major cause of death. The most recent community assessment available from 2010 showed that St. Louis City had 718 deaths from heart disease (rate=228 per 100,000) and 2,432 deaths (rate=181 per 100,000) in St. Louis County (Department of Health and Human Services, Missouri Information for Community Assessment [DHHS/MICA], 2013). Lack of access to a primary care provider and affordable healthcare further exacerbate heart disease incidence and prevalence in Missouri. For example, rural Missourians frequently lack access to primary care providers and approximately 193,430 Missourians fall within an insurance coverage gap due to eligibility requirements in the Affordable Care Act and Missouri Medicaid ("How will the Uninsured," 2014; Missouri Department of Health and Senior Services [MODHSS], 2011; “The Coverage Gap," 2014).

The AHA reported 670,000 new cases of HF diagnosed annually with projected health care costs exceeding 32 billion dollars in 2013 and 70 billion dollars by 2030, makingHF the most costly cardiovascular illness in the United States (Go et al., 2013; Rogers et al., 2011, 2012). Nearly half of those diagnosed with HF survive less than 5 years from initial diagnosis and among NYHA class IV, $60 \%$ to $80 \%$ have a less than2-year mortality rate (Roger et al., 2012; Stevenson et al., 1995).

The societal cost of treating and ensuring a quality of life to persons with $\mathrm{HF}$ is an expensive proposition with a limited duration of successful outcomes for many. Heidenrich and coworkers (2011) justify that quality of life measures affirm value,yet spending inefficiencies must be analyzed. Quality adjusted life years (QALYs) and disability of life years (DALYs) measuresare being utilized to evaluate the economic value of treatment strategies, their cost effectiveness, and resource allocation (Alehagen, Rahmqvist, Paulsson, \& Levin, 2008).Alehagen, Rahmqvist,Paulsson, and Levin (2008) demonstrated that HFpatients with NYHA classes I - IV had QALY weights of $0.77,0.68,0.61$, and 0.50 , respectively (QALY scores range from .10 to 1.0), where an increase in symptomologysignificantly impacted the patients'perceived quality of life. Skerrett (2012) emphasized thatdiseases with risk factors such as high blood pressure orhigh cholesterol levels, rise in diabetes, being overweight and underactive are largely preventable.Furthermore, hospitals with high readmission rates for adiagnosis such as HF receive less payor reimbursement, tied to a quality expectation that health providers need improved strategies to clarify, better manage, and prevent hospital readmissions (Koekkoek, Bayley, Brown, \&Rustvold, 2011). Locally, in St. Louis City there were 788 preventable congestive HF hospitalizations (rate $=25.7$ per 10,000) and 1,115 (rate=10.7 per 10,000) in St. Louis County costing $\$ 6,274,213.6$ and $\$ 8,877,853$, respectively, with length of stay at 4.1 days and expenses per inpatient day at \$1942 (DHHS/MICA, 2013; "Hospital adjusted,” 2012; "Reduced Heart Failure,” n.d.).

\section{Risk Reductionin the Area of Heart Disease}

Another opportunity for providers to help patients with knowledge acquisition rests in the area of heart disease risk reduction. As noted above, Missouri has a substantial number of people who experience death, disability, and expense tied to heart disease and stroke. Yet a framework exists to modify risk factors that advance prevention and intervention. According to the MODHSS (2007), adults in Missouri are at equal risk for heart disease and stroke when compared to nationwide averages on obesity (Missourians=63.3, nationwide $=62.9 \%)$; physical activity $(51.2 \%, 50.5 \%)$;total blood cholesterol levels $(39.5 \%, 37.6 \%)$; and blood pressures $(29.4 \%, 27.8 \%)$; yet notably above national averages related to smokingcigarettes $(24.5 \%$, $19.8 \%)$.These high risk factors cause changes in the heart that lead to an increased risk for heart attack, HF, and stroke, and are largely preventable with diet, activity, smoking cessation, and other behavioral adaptations.

Modifiable risk factors for heart disease and stroke such as high blood pressure and cholesterol, cigarette smoking, diabetes, poor diet and inactivity, and overweight and obesityhave the potential to limit functional status, productivity, and quality of life (US Department of Health and Human Services [USDHHS], 2013). Formulating the best plan of care is critical to the partnership between the person withHF and the HF healthcare team. Sharing knowledge through well established, commonly usedguidelines such as AHA clinical practice and patient education guidelines may help the person with HF better understand and accept a plan of care that could improve patient outcomes. 


\section{Acute Care Experience}

When a HF patient requires acute hospitalization, it is never a good sign. Hospitalization often marks the progression of the disease and the need for extensive testing, treatment recalibration, and further changes in lifestyle which impacts the quality of life. The acute care hospitalization is a vulnerable time in the trajectory of someone with HF.

Within the acute care experience, the HFteam urgently unites:extensive testing and imaging are performed of the heart, kidneys, and lungs;symptoms are stabilized;and progression of the disease or further functional impairment is evaluated. Patients with HFoften haveincreased anxiety, or depression, overfeelings about death. These feelings may affect patients'motivation to modify their lifestyles and desire for remaining autonomous in performingself-care (Gallacher, May, Montori, \&Mair, 2001; Stromberg, 2005).Episodic treatment or frequent hospitalizations is inadequate care for patients with HF (Moser \& Mann, 2002). Education and counseling supporting prescribed medication adherence and the reporting of symptom decompensation or escalation are essential aspects of disease management. Self-care management is when one chooses to perform activities that promote health maintenance and physiological stability. Care management may have to shift to others in the family when HF progresses (Chen, Yehle, Plake, Murawski, \& Mason, 2011).Goals for self-care management require a high level of accountability from the person with HF at their capacity level. The partnership among the HCP, the person with HF, and the family, is intense during the acute care experience. Knowledgeis sharedon medication, symptom management, and goals of care in order to help patients adapt to their condition and assumeself-care at an appropriate/negotiated level of engagement.

\section{Statement of the Problem}

Heart failure is a complex disease syndrome that impacts the patients, their families, healthcare providers and institutions, and society at large. The high incidence and prevalence of HF noted is compounded by environmental conditions, socio-cultural norms, personal preferences, and hereditary factors. Once diagnosed, quality of life and life expectancy is likely shortened. The disease trajectory itself is marked with points of anxiety, family role adaptation, and shifting limits in self-care capacity (Gallacher et al., 2011; Matlock, Nowles, \& Beckelman, 2010; Thornhill, Lyons, Nouwen, \& Lip, 2008; Van der Wal et al., 2007). There are many interventional approaches available to treat $\mathrm{HF}$ patients including clinical procedures, medications, dietary practices, and exercise aligned with the capacity of the heart muscle to accommodate activity(Fredericks,Beanlands, Spalding, \&Da Silva, 2010; Hallerbach et al., 2008). For these reasons and based on the high morbidity and mortality of this disease, interventions that include patient education impact the trajectory of this disease. As stated, lifestyle adaptation, and symptomology recognition is essential (Lofvenmark, Saboonchi, Edner, Billing, \&Mattisson, 2012). Sign and symptom recognition is critical, as these are the hallmarks for interaction with the HCPs providing clinical support. If ignored, hospitalization is likely necessary for reassessment and recalibration of treatment options. This reassessment and recalibration creates yet another critical opportunity for patient education. In spite of the importance of patient education, very little is known about who on the health care team provides this education, what content is covered, and how patients and families experienceacquiring HF content.

In this study, naturalistic inquiry is used to collect and analyze data on how patients and families acquire HF knowledge and the substance and comprehensiveness of education as a clinical intervention in the acute care setting. As an observer, the researcher adopted apedagogical posture that includes observing, analyzing, and reflecting on provider and patient interactions. The datacollection and analysis process is one with emerging insights which moves from the problem determined by the study to a purposive one seeking typical or divergent data to maximize the range of data available within the context (Erlandson, Harris, Skipper, \& Allen, 1993, p. 148). In naturalistic inquiry, this interactive process of data collection and analysis leads to the formation of a gestalt at the conclusion of the study.

\section{Purpose of the Study}

The purpose of this study was to determine what HF information the patient receives in the acute care setting through the patient - HCP exchange.Observational data were analyzed and compared with AHA guidelines for congruency, gaps, or where content extends beyond the AHA guidelines. The aims of this study wereto: (1) determine what AHA Guidelines are utilized in the hospital setting; (2) what HF instruction went beyond the AHA Guidelines; and (3) determine what experts felt was important instruction in the acute care stay. Data collection lead to patterns, themes, and experience recognition to address the following questions:

- 1a. How much total time was spent on AHA topic related instruction?

- 1 b. Which AHA topics and content were covered?

- 1c. How much time was spent on each AHA topic related instruction?

- $1 \mathrm{~d}$. Which HCPs provided the instruction for each AHA topic? 
- 2.a What content fell outside of the AHA Guidelines?

- $2 b$. Which HCPs provided HF instruction outside the guidelines?

- 2c. How much time was spent on this HF teaching?

- 3a. Where was the congruence between HF Guidelines and expert recommendations/instruction?

- $3 \mathrm{~b}$. Where was there incongruence between the HF Guidelines and expert recommendations/instruction?

For the purposes of this study, expert providers are defined as those HCPs who have the responsibility for directing/leading the HF team and who are experts in HF care management.

\section{Delimitations and Limitations of the Study}

A delimitation of the study is that the unit of analysis deliberated on educational opportunities between the patient-provider exchanges and did not include the patient-family dyad. An alternative setting may offer more intense instruction andeducational opportunities for the patient-family dyad.A limitation of this study is that the findings are not generalizable. Traditional research is focused on random sampling and generalizable data. Yet, in naturalistic inquiry, thick descriptions give the reader a sense of what it is like to be in the context, and purposive sampling is the process that provides this rich detail (Erlandson et al., 1993). Generalizability limitations must be acknowledged. Concurrently, the different purposes and strengths of naturalistic inquiry allow the discovery and exploration of the participants' perspectives without predetermined assumptions. These findings may be judged as transferrable from one context to the next.

\section{Theoretical and Conceptual Foundation}

Mastery Learning Theory (Guskey, 1985) posits that people learn information at different speeds and may require one or more exposures to material in order to master it. Learning mastery is the process of breaking down information into smaller units and then building on this knowledge in a logical progression. Education leads to knowledge, however, self-efficacy, the belief that one can achieve his or her goals, plays an important role as a mediating factor. Bandura's (1986)social cognitive theory emphasized learning mastery andselfefficacy.According to Bandura, the four essential components of learning areattention, retention, reproduction, and motivation.The effort one exerts in goal attainment/mastery positively affects self-efficacy.As a result, the reciprocal processes between the cognitive (self-efficacy), behavioral (task performance), and environmental (resources)domains within a context allow for successive learning to occur and increase opportunities for learning and mastery. Enhanced education interventions need tofocus on improving self efficacywith cognitive behavioral approaches, i.e. learning activities that strengthen confidence and reach self-care goals.

Baker and colleagues (2011) found that learning goals or"Teach to Goal" interventions for people with $\mathrm{HF}$, paired with the learning mastery formula, promotes learning goals, facilitates future learning, and prevents deterioration or escalation of symptoms and hospitalization. The AHA provides well-established"Teach to Goal"guidelines on HF and supports this patient education to advance patients' capacity to manage their HF post discharge. Lack of patient knowledge to manage complex clinical treatment plans is a reason for noncompliance (Murray et al., 2007).

Baker (2011) states teaching self-care management should be a fundamental educational goal.Efforts to educate HF patients on self-care management regimes, such as recognizing symptoms of worsening HF, restricting fluids and dietary salt, monitoring weight, adhering to medications, exercising, restricting alcohol, and smoking cessation, have shown improved health outcomes, patient compliance, and reduced costs (Baker et al., 2011; Driscoll, Davidson, Clark, Huang, \& Aho, 2009). The acute care experience becomes an essential opportunity to educate and validate the patient's self-care management abilities and to reinforce health maintenance practices.Ideally these principles are illuminated by the healthcare team during the acute care experience.

Patients with HF continue to be confused with their illness and how to manage it (Rogers et al., 2002). Patients with HFhave very complex treatment regimens and are most vulnerable for hospital readmissions (Krumholz et al., 1997). McMurray and Stewart (2000) indicatedHF is becoming the most common manifestation of heart disease and contributor to death in the US, yet there may be missed opportunities toteachHF patients about their chronic condition. Formulating the best plan of care is vital for optimal health outcomes, and utilizing theory (mastery learning theory, social learning theory) and AHA guidelines developed to help the patient with self-care management skills is key to successful implementation.

\section{Summary}

This study noted content and context for how patients and their families acquire and experience HF education as an important intervention offered to patients through patient - provider exchanges. The observations were made on HF patients during an acute care stay using evidence based AHA guidelines as a baseline. Very little is known about who on the health care team provides education, what teaching methods are 
used, what content is covered, and how patients and families respond to knowing this content to be helpful, once acquired. Exploring this patient-provider exchange in the hospital setting using a qualitative, naturalistic approach may provide a subsequent clarification of teaching - learning processes or experiences that could contribute to care improvement, at both a patient and organizational level, including readmissions.

While there are many elements that must be considered in managing heart disease, there is compelling evidence for utilizing anapproach that includesevidence based guidelines and strategies, such as these developed by the AHA, that can improvethe delivery of patient education. Successful management of HF requires a high level of self-care with open patient - provider communication.Exploring this patient-provider exchange in the acute care experience may provide a subsequent clarification of processes or experiences and contribute to improving quality of care, providing accessible information related to symptoms of $\mathrm{HF}$, and preventing readmissions.Currently there is a paucity of research from the qualitative perspective on AHA instruction shared during the patient-provider exchange during an acute care stay.

\section{Definition of Terms}

Culture: The "learned, shared, and transmitted values, beliefs, norms, and lifeways of a particular culture that guides thinking, decisions, and actions in patterned ways and often intergenerationally" (Leininger\& McFarland, 2006, p. 13).

Cultural competence: The awareness of one's own thoughts, sensations, and environment without allowing inappropriate influence on patients of another culture; demonstration of knowledge and understanding of the patient's culture; acceptance and respect for cultural differences; and, adaptation of patient care and practices that are respectful of the patient's culture (Suh, 2004).

Health literacy: The ability to read, understand, and act on health information (Institute of Medicine [IOM], 2004).

Heart failure: A condition in which the heart is not able to pump enough blood to meet the body's needs. Either the heart is not able to fill with enough blood or the heart is unable to pump blood to the rest of the body with enough force. Some patients have both problems (Heart failure, 2012).

Inpatient stay/hospitalization: Inpatient physician orders were written on the medical record at the time of admission.

Expert providers: Those HCPs who have responsibility for directing/leading the HF team and who are experts in HF care management.

Self-care: "Specific behaviors that [patients] initiate and perform on their own behalf with the intention of improving health, preventing disease, or maintaining their well-being" (Evangelista \&Shinnick, 2008, p. 2).

Self-management: The patient monitors symptoms, makes decisions, and evaluates the impact of the decisions (Evangelista \&Shinnick, 2008, p. 3).

Self-efficacy: The belief that one can achieve his or her goals (Bandura, 1986).

Socioeconomic status: “An individual's or group's position within a hierarchical social structure. Socioeconomic status depends on a combination of variables, including occupation, education, income, wealth, and place of residence. Sociologists often use socioeconomic status as a means of predicting behavior" ("SES," n.d., para. 1).

For the purposes of this study, the following acronyms were used: NP, nurse practitioner; and, NE, nurse educator.

\section{Organization of Remaining Chapters}

Chapter Two provides an overview of the existing literature on HF and the experience of knowledge acquisition. The research methods used to conduct this study are described in Chapter Three. Chapter Four contains the research findings. Chapter Five compares the findings to the related literature and identifies the implications and conclusions of this study.

\section{LITERATURE REVIEW}

\section{Review of Relevant Research Literature}

Heart failure is a syndrome that is marked by a progressively complex clinical state that requires close relationships and care coordination with HCPs. A critical aspect of care is an intervention that centers on patient education, as with this disease state symptom management is crucial to safety and comfort, care in a high cost environment,and avoiding disease progression. Without content knowledge of these factors, patients are at risk. As noted in Chapter One, the purpose of this study is to identify:(1) what AHA Guidelines are utilized in the hospital setting; (2) what HF instruction went beyond the AHA Guidelines; and (3) what experts felt was important instruction in the acute care stay. Four themes emerged from a review ofthe relevant research. These themes that frame this study are presented in four categories: heart failure education; the patient experience; coordination of care; and, heart failure guidelines. For the purpose of this chapter,HF education interventions 
are summarized first. Second, the patient experience is presented. Next, the coordination of care is reviewed. Finally, AHA guidelines are detailed.

\section{Heart Failure Education}

According to Fowler (2012), HF education for providers to consider with their patients' needs to address theself-care management categories of medication management, activity/exercise, low sodium diet, signs and symptoms of decompensation, and pain management. Patient education is a precursor to a patient's ability to perform self-care andtake action; therefore, healthcare providers must acquire and tailor health related knowledge as part of their clinical routine (Boyde et al., 2013; Clark, et al., 1998).Tailoring the right educational method to the patient experience matters for it to impact self-care knowledge, self-care behavior, and symptom management as noted in a systematic review of HF education literature (Fredericks, et al., 2010). The most effective methods of patient education included the introduction of individual content, use of videos and printed handouts, a one-to-one delivery of education, and reinforcement with repeated sessions. Additionally, one-onone or group education approaches improved patient confidence andself-efficacy and helped with adherence to self-care behaviors for HF patients (Paradis, Cossette, Frasure-Smith, Heppell, \&Guertin, 2010; Yehle\&Plake, 2010). Effective HF patient education requires healthcare providers to be flexible and resourceful in their teaching approaches.

At some point in time, the progression of HF is inevitable. Support networks or family caregivers who will eventually play a more dominant role in HF management must be included in education. The sickest patients with heart disease may not be able to self-manage their symptoms. Beckelman et al. (2011) and Rosland (2011) identified that early involvement of family caregivers helped prepare the patient for the adjustments to ADL and clinical management as HF progressed. This involvement was more substantial for patients with lower literacy levels, four or more co-morbid conditions, and symptoms of depression.Lofvenmark et al. (2012) noted that multidisciplinary approaches to patient education interventionsfor family caregivers showed no significant effects on the anxiety, depression, and quality of life for family caregivers of HF patients. They suggest that HCPs who dialogue with their patient and family caregivers contribute a level of social supports that can "lower levels of anxiety and depression" (p.123).

Having generalized rather than disease-specific knowledge may impact the nurse's role as educators in HF management (Kalogirou, Lambrinou, Middleton, \&Sourtzi, 2013). Three studies examined 300 nurses who received a 20-item HF knowledge survey after receiving HF education aimed at strengthening their content knowledge on HF (Albert et al., 2002; Washburn, et al., 2005; Willette et al., 2007).Even with reinforced HF education, these nurses scored low on knowledge of HF self-management principles such as the importance of a low sodium diet, restricted fluids, weight monitoring, reporting worsening signs and symptoms, medication adherence, and exercise for patients. Conversely, Fowler (2012) found HF education improved nurse knowledge on HF self-management principles. With access to disease-specific patient education resources, nurses are more likely to carry out the nursing function of patient education (Chick, Negley, Sievers, \&Tammel, 2012). Next, in a systematic review of HF education programs, 35 random control trials showed nurses to be the most frequent educators in hospitals, clinics, and home; and, verbal, hand written, and printed handouts were the most common teaching methods employed (Boren, Wakefield, Gunlock, \& Wakefield, 2009). Nurses must possess HF teaching materials and follow protocols in order that their HF patients may possess knowledge and guidelines that facilitate self-management of HF.

Unlike the Lofvenmark study, Roncalli et al. (2009) found that multidisciplinary teaching is effective. Patient education on self-care management has shown benefits of increased self-management knowledge and delayed progression of disease.Roncalli et al. (2009) found sustained improvements in knowledge level and selfcare management in patients with HF whoreceived educational interventions from multiple disciplines. The goal was to improve patients' HF knowledge of self-care behaviors and improved patients' desire to seek more knowledge about their disease.Patients surveyed scored 9.7/20 before themultidisciplinary intervention and 16.3/20 and 16.6/20 in the 1st and 4th quarter respectively after the intervention (Roncalli et al., 2009). In a pilot study using a pre- and post-test design, a convenience sample of 38 patients with congestive HF received a multimedia educational intervention to measure changes in HF knowledge and self-care behaviors (Boyde et al., 2013). Two surveys showed significant results on HF knowledge $(p<0.0001)$ and self-management scores specific to maintenance $(\mathrm{p}=0.027)$, management $(\mathrm{p}=0.0001)$, confidence $(\mathrm{p}=0.051)$. Interventions that include a multimedia, multidisciplinary approach have shown reduced confusion regarding symptom management, improved outcomes, and reduced or prevented hospital admissions and health care costs.

HCPs play a primary role for the diagnosis, treatment, and patient education provided to HF patients. Reinforcing HF content to guide appropriate self-management changes has been shown to help patients and their caregivers adapt to this chronic condition. Finally, HCPs should make every effort to seek feedback from the patient or family caregiver in order to identify barriers to self-management or help them adjust to the current priorities of care. 


\section{Patient Experience}

Understanding the patient experience, or perspective, provides a better chance for improving quality of care, and education framed through this lens adds to tailored educational approaches (Erlandson et al., 1993). In one qualitative study, 27 patients were interviewed who had been recently hospitalized (Rogers et al., 2000). The study uncovered varying degrees of knowledge on the cause of $\mathrm{HF}$ and prognosis, and descriptions of what inhibited good communication: doctors did not want to provide too much information (patients perceived clinicians as believing the patient too stupid to understand); transportation difficulties to keep follow-up appointments; and the manifestation of confusion and short term memory loss as part of disease symptomology (Rogers et al., 2000).In a critical synthesis of seven articles on the experience of HF patients, the following outcomes were noted: patient confusion about medications (not understanding the purpose of drug therapy, quantity and combination of medicines, symptoms versus side effects of drugs); the ability of the provider to transfer knowledge which could relieve anxiety, change illness beliefs, and improve compliance; and patient adjustment to physical and social limitations, extensive treatments, and, active/passive decision making (Duhamel, Dupuis, Reidy, \&Nadon, 2007; Hain, Tappen, Diaz, \&Ouslander, 2012; Gallacher et al., 2011; Matlock et al., 2010; Rogers et al., 2002; Thornhill et al., 2008; Van der Wal et al., 2007). This broad base of interrelated factors allows providers to target interventions that are patient- rather than disease-centered.

As HF knowledge and treatment options expand, so have the educational demands placed on patients expanded in complexity related to learning and managing health information. For a patient who survived a myocardial infarction in the 1960s, the patient was discharged from the hospital without education; in the 1990s, that same patient would have been placed on a protocol of "aspirin, a beta blocker, an angiotensin-converting enzyme inhibitor, and possibly a low salt and low cholesterol diet and medications to control hypertension, diabetes, and hypercholesterolemia" (Baker, Parker, Williams, \& Scott, 1998, p. 791).Patients who are unable to learn this regimen or carry out other health instructions for chronic illnesses are vulnerable. Patients with lower literacy have the greatest burden when it comes to comprehendinghealth-related reading, which can worsen health outcomes. It is the single best predictor of how patients will acquire knowledge about their chronic illness and health status (Campinha-Bacote, 2007).

An estimated 90 million people have difficulties understanding health information:read at the fifth grade level or below, or have limited English proficiency (Parker \& Ratzan, 2010). Inadequate health literacy imposes barriers to better self-care ability, increasing the risk for poor outcomes and preventable hospitalizations. Understanding and applying knowledge to specific situations, such a shortness of breath or increased edema, has a direct relationship to health status. Peterson's (2012) studyused a three question health literacy assessment and results revealed a significant association between low health literacy and higher allcause mortality in patients with HF. Patients with low literacy $(17.5 \%)$ were more likely to be elderly, have lower socioeconomic status (SES), and have at least a high school education. Older HF patients' health literacy could be inaccurately categorized by HCPs when cognitive deficits, such as confusion or short term memory loss, are manifested (Morrow et al., 2006; Robinson et al., 2011; Rogers et al., 2000).HCPs must not rely on a gut feeling or use the last grade completed to determine a patient's health literacy as these appraisals may overestimate or underestimate the patient's literacy (Barrett, Puryear, \&Westpheling, 2008; Baker et al., 2007). Utilizing a strategy that identifies the patient's learning preferences or tailoring HF teaching to meet the selfcare needs of patients with lower levels of health literacy or cognitive abilities ultimately helps those most in need of clear, accurate instruction.

Cultural considerations such as openness, mutual respect, inclusiveness, responsiveness, and understanding one's role were found to be important fundamentals in the delivery of culturally competent healthcare (Purden, 2005).In one study, race concordance between provider and patient or a perceived patient and provider similarity showed high ratings of trust, intentions to follow treatment plans, and overall patient satisfaction (Street et al., 2008).

It has been well documented that minority groups experience a disproportionate burden of chronic disease and differences in the care received (IOM, 2004; Smedley, Stith, \& Nelson, 2003). HF illness beliefs were examined noting that beliefs about HF were less accurate in African Americans with lower education levels compared to non-Hispanic Caucasians with higher education levels (Albert, Trochelman, Meyer, Nutter, 2009). Inaccurate beliefs may be uncovered and replaced with HF-specific information during the hospital admission. Reasons minorities receive lower quality health care were identified as: fragmented healthcare systems, cultural and linguistic barriers, incentivized cost control, and conscious or unconscious discrimination and use of stereotypes. A study related to confidence in self-care management and race/ethnicity reported that Hispanic (36.5\%) and Black (30.7\%) patients had lower confidence compared to White (16\%) patients; and low SES and poor health were explained as a potential "mechanism by which some disparities work" (Blustein, Valentine, Mead, \& Regenstein, 2009, p. 927). With society becoming increasingly diverse in its cultural, 
ethnic, linguistic, and religious composition, providers of care and healthcare organizations nationwide will need to respond in ways that optimize the patients understanding of their illness, treatment, and desired outcomes (Kumagai\&Lypson, 2009).HCPs should focus onteaching-learning strategies compatible with HF patients' cultural needsto provide culturally competent care rather than disconnected teaching interventions.

\section{Coordination of Care}

Nurses, cardiologists, primary care physicians, and other health care professionalsmust collaborate to offer individualized patient centered care tobenefit HF patients and to also demonstrate cost effective and efficient ways of delivering education (Pugh et al., 1999). Nearly 15 years ago a collaborative care or a partnerin-care model was tested as a multidisciplinary strategy for discharge planning, disease management, and patient education based on three factors: medication, symptoms, and diet (Pugh et al., 1999). A nurse case manager closely monitored 200 patients age 65 years or older with HF in the hospital and home settings for 6 months, regularly reviewing patient progress and providing symptom management, weight monitoring, diet, and exercise education reinforcement. The study found that close monitoring and communication between the provider and patient helped reduce HF symptoms (dyspnea), improved outcomes with pulmonary rehabilitation, and medication adjustments after discharge.

In a randomized control trial, stage $4 \mathrm{HF}$ patients who received multidisciplinary care (MDC) had the lowest rate of mortality events (7.8\%) compared to $25.5 \%$ mortality events in the routine care (RC) group (McDonald et al., 2002). Both groups were provided with protocol driven treatment and specialist-led care while hospitalized; however, when discharged the MDC group received close inpatient and outpatient education with telephonic and clinic follow-up. The study indicated that most of the MDC was provided within the hospital setting. Patients who were provided with MDC after hospital discharge had lowered hospitalization rates, improved satisfaction (Reigelet al., 2002), reduced readmissions or death, and, reduced readmission costs (Stewart \& Horowitz, 2002). The evidence showed positive outcomes with nurse-led HF management programs and indicated thatusual care is not adequate for HF patients.

Failure to coordinate care andidentify safety risks, as well as a lack of provider advice on chronic disease management, are global issues (Schoen et al., 2005).No one country "leads or lags" (p. 509) within the aforementioned domains. This global experience makes HF patients at risk in many models of health systems.

\section{Heart Failure Guidelines}

In 2005, American College of Cardiology (ACC) and the American Heart Association (AHA) published revised evidence based guidelines that included both pharmacological and non-pharmacological interventions for the prevention, diagnosis, and management of heart failure (Hunt, 2005). Adoption of HF guidelines is slow and inconsistently applied, affecting quality of care and patient outcomes (Lee et al., 2005; Williams, Schmaltz, Morton, Koss, \& Loeb, 2005). Sreih (2004) found improved utilization of spironolactone in patients with systolic dysfunction from $0 \%$ to $40 \%$ after adding clinical decision alerts within a physician order entry system and providing an education intervention for raising awareness on HF guidelines. Utilization of angiotensin converting enzymes (ACE) inhibitors/angiotensin receptor blockers (ARBs) and beta blockers did not significantly fluctuate after the education intervention. However, documentation of contraindications for ACE inhibitors/ARBs and beta blockers substantially improved.

Fonarow et al. (2010) investigated the treatment gap between HF guidelines and clinical practice extensively, showing improvements on quality measures after the introduction of clinical decision support tools, chart audits, and feedback. A meta-analysis of 18 studies related to discharge planning and proper discharge support showed reduced all-cause mortality, length of stay, and improved quality of life (Phillips et al., 2004). Further, a study related to patient education with standardized instruction for 60 minutes prior to discharge showed improved outcomes, increased adherence to plan of care, and reduced healthcare costs (Koelling et al., 2005). The evidence supports that HF guidelines, when used, can make a substantive difference. Patient education is an important intervention in these recognized guidelines.

\section{Summary}

The results of this literature review covered four key themes includingHF education, the patient experience, coordination of care, and HF guidelines. TheHCP and HF patient encounter is more than just a clinical assessment and treatment of an acute or chronic condition. Providing HF education that follows patient sensitive guidelines and inquires about sensitive issues such as social support, SES, literacy, and cultural beliefs related to one's race/ethnicity, is essential. If the plan of care is not being communicated effectively by the HCP or understood and carried out by the HF patient, society pays the price with poor health outcomes and increasing health care costs.

In the hospital setting, a multidisciplinary team can provide the HFpatient education with individualized patient centered recommendations for treatment and outpatient follow-up care. Lack of provider 
knowledge on HF education principles or guidelines, ignoring or not knowing how to handle different patient experiences, or missed opportunities to ensure transitional outpatient follow up care, may stand in the way of optimal patient care and health outcomes. In this study, a naturalistic approach is utilized to better understand the dynamics involved during the care, treatment, and education of the hospitalized HF patient by a multidisciplinary team.

The multidisciplinary team influences the care received and outcomes within the hospital setting and beyond. Knowledge on themes identified here may help to understand efficiency and effectiveness of the care provided or missed opportunities therein. How the multidisciplinary team responds to the identified or subtle needs of the HF patient during an inpatient hospitalization has not been well understood and is central to this research study.

\section{METHODOLOGY}

\section{Methodology}

The research questions posed in Chapter One acknowledge the importance of health education provided by the health care team for HF patients in the acute care setting. Yet nothing has been documented or studied to date that addresses:(1) what AHA Guidelines are utilized in the hospital setting; (2) what HF instruction goes beyond the AHA Guidelines; and (3) what experts felt was important instruction in the acute care stay. Using a constructivist, naturalistic inquiry approach, all relevant variables pertaining to the phenomenon can be sought (Appleton \& King, 1997; Erlandson et al., 1993; Lincoln \&Guba, 1985). This study uncovers important dimensions, patterns, and complexities of HF patient-provider interactions as a social phenomenon experienced within an acute care hospital setting.

This chapter explainsthe philosophical underpinnings of the naturalistic inquiry research paradigm and the role of the researcher. Additionally it coversa description of the data collection procedures utilized for participant selection, observation technique of patient-provider exchanges, outcomes reporting, and credibility and dependability of data verification.

\section{Assumptions, Naturalistic Inquiry, and the Research Questions}

In order to better understand the world we live in, constructivism or naturalistic inquiry initially asksthree basic questions: 1) What is the form and nature of reality and what can be known about it? 2) What is the nature of the relationship between the researcher and what can be known? and, 3) How should the researcher go about gathering knowledge to answer the research question? (Appleton \& King, 1997). It is assumed that a disciplined inquiry can proceed towards the why and how of human behavior, not just the what, where, and when, to make explicit what is implicit and intuitive.

In doing naturalistic inquiry, there are five axioms that guide and support the researcher. Lincoln and Guba (1985) present these axioms as: 1) the nature of reality (ontology) where realities are multiple and constructed; 2) the relationship of the knower to the known (epistemology) where the knower and the known are interactive, inseparable; 3 ) the possibility of generalization where only time and context bound research aims are possible; 4) the possibility of causal linkages where entities are in a state of mutual simultaneous shaping making it impossible to distinguish cause and effect; and 5) the role of values where inquiry is value bound instead of quantified or data derived.

Other research methods were considered for this study including prompted questionnaires to gather and analyze data. After a review of the current methodology literature, it was determined that these objective approaches imposed a distance between the inquirer and the experience and behaviors of the subjects that is of prime importance. Furthermore, a paucity of research exists on the topic of what HF instructional content is delivered toHF patientsin the acute care setting which might then lead to the "why and how" noted above.By exploring the intricate HCP and HF patient relationship, clarifying processes and experiences of shared content knowledge with greater precision, this study frames an unknown perspective on HF education. Based on the critical importance of the context, overview of the situation, and the research questions, the naturalistic inquiry was selected as the best fit for this study.

To answer the research questions a qualitative approach, plausible in naturalistic studies, was necessary. A qualitative approach facilitated an in depth and detailed examination of the provider-patient educational experience. Quantitative data collected purely for descriptive purposes and not as outcome measures, permitted comparisons and contrastsbased on predetermined categories of HF education, established through evidence by the AHA, a national disease oriented specialty organization, as essential for patient management of HF. Finally, experts were surveyed to capture field-based reality aimed at HF education. This strategy is necessary to gain an understanding of a phenomenon (Haase \& Myers, 1988).

When the intent of a study is to uncover patterns, subjective experiences, and the nature of meaning behind complex phenomena, the naturalistic researcher is supported by the qualitative approach. An advantage of reporting from the naturalistic standpoint is the researcher being vicariously placed in contact with the 
context, allowing for their comprehension to be shaped by thick descriptions and led into the complexities of how the real world affects human behavior.

This researchercollected data from observations of HCP and HF patientinteractionsas the primary data gathering method (See Appendix A). After theobservations were concluded, asurvey was presented to expert HF HCPsincluding cardiologists, internal medicine physicians, and nurse practitioners (See Appendix B).The survey quiered what these experts believed to be critical HF education to patients in an acute care hospital.Thedata collected wasanalyzedand coded, then wascomparedand contrasted in a model of ideal versus actual HF education provided against what expert providers considerednecessary(See Appendix C). This analysisrevealed where there is agreement, where there is content that extends beyond or is different from the recommended national standards (AHA), and where gaps exist. What resulted is a deeper and multi-perspective capture of HF education and how patients and providers interact to create knowledge application.

The data were triangulated. A broad definition of triangulation iswhen a researchercollects information within the field of study about the same event from different points of view and methods (Creswell \& Clark, 2007; Erlandson et al., 1993; Patton, 1999) and compares and contrasts results. Fieldwork includes triangulation, by searching for disconfirming evidence, reflexivity, prolonged engagement, member checking, collaboration, audit trail, and peer debriefing (Marshall \&Rossman, 2011). Lincoln and Guba (1985) offered the aforementioned as acheck list of items to ensure credibility, dependability, and transferability. These three qualitative criteria ensure trustworthiness and credibility of research practice comparable to the quantitative canons of reliability, validity, objectivity, and generalizability.

\section{The Research Setting}

Naturalistic inquiry requires substantial observation and interaction with participants in the natural environment or in this case, an acute care hospital. The setting and the five factors for its selection was identified and justified: appropriateness, access, ethics, immediate risk, and personal consequences (Lofland\&Lofland, 1995).

Appropriateness of the setting. This study utilized observations, field journaling, artifacts, and a survey ofexpert providers within the hospital setting. Anacademic hospital setting with four units of HF patients assured the researcher access tosufficient numbers of patients with $\mathrm{HF}$ as a primary diagnosis. Academic health teams are multiprofessional, so to capture a dynamic between HCPs was useful. A purposive sample of HF patients who had progressed to stage 3 or 4 was ensured in this setting. Guba and Lincoln (1985) expressed the need for providing a broad range of information possibilities and a full array of multiple realities. This larger hospitals was structured with specialized teams of health care providers, services, and treatments for HF patients with care taking place on multiple cardiology units.

Access. Access to the research site was eased as the researcher has work experience in this academic hospital setting. Knowledge of the setting and aspects of the care delivery gave the researcher a sense that data collection would be robust with its rich mix of people and plethora of interactions. Nonetheless, the familiarity of the researcher to the setting triggered concern such as the researcher being seen by HCPs in a researcher role rather than a familiar work role, the potential for an ethical dilemma such as the potential for uncovering damaging knowledge, and intense interaction during research and closure (Marshall \&Rossman, 2011). Yet the positive aspects of being in a familiar setting including easy access to participants, reduced data collection time, and a subjective understanding of phenomenon and participants outweighed the negative.

Ethics. The study was submitted to an Institutional Review Board (IRB) for the Protection of Human Subjects and approved prior to implementation, ensuring the protection of research participants'rights. The study had no invasive procedures linked to it and all participants recruited werebetween 50 and 80 years of age. Protection and respect for the privacy of all participants required special precautions. The enrollment and consent process was completed by the researcher for two populations, the patient and HCP participants. In the first, the primary investigator explained the study procedures to potential patient participants:each patient was provided enough time to enroll so they couldthoroughly consider what their participation meant; no nurse managers were present during the enrollment or consent process in order to minimize coercion. Once the researcher explained the purpose, study procedure, and risks to participants, written informed consent was obtained from each. Given that both patients and HCPs had to mutually consent, if the patient agreed to participate but the caregiver declined, the primary investigator withdrew both from the study. The following was explained to potential patient participants: (1) patient and HCP interactions would beobserved immediately each contiguous day from admission through discharge;(2) the researcher wouldremain outside the patient's room and only enter the patient's room when a HCP enteredin order to observe interactions; (3) the researcher would take field notes and collecteducational materials given to patient participants; and (4) that each patient would be assigned an identification number to maintain anonymity. 
In consenting the HCPs, the researcher notified a manager, director, or chief from each health care discipline at first requested their permission to conduct the study. A list of names and emails of HCPs who work on involved nursing units was then obtained. The researcher thenemailed each HCP no more than twiceto request their consent and were enrolled when the email was returned. Whether or not the HCP participated did not impact the individual employment standing or evaluation. The consent process included:(1) that HCP and HF patient interactions would be observed. In the event that a written consent was not secured, the IRB approved an alternative verbal consent to present the terms of participation, avoiding unnecessary delay of care; (2) participation was voluntary and witheither an emailed or verbal consent to participate - the names would be coded for anonymity entered on a list of consented participants; (3) any unknown HCPs followed the above process, i.e. consultants; (4) once consented, the researcher would observe theHCP and HF patient interactions, take field notes, collect artifacts, and copy educational materials given to the patient; and, (5) each provider of care was assigned an identification number as a form of protecting their identity.

Expert HCPs were: (1) offered a survey at the conclusion of the field review, who met the criteria for being an expert, this survey had beenoutlined within the initial consent;and (2) if the provider chose not to take the survey, the researcher thanked him/her for their time with no undue influence.

Truthful or full disclosure was included in the study that informed participants of their right to refuse, researcher's responsibilities, and the likely risks and benefits (Polit \&Hungler, 1999). Yet,a conscious decision was made to focus on patient and HCP interactions, a subset of which was the domain of patient and HCP HF information. Specific content from the AHA Guidelines was not revealed. Not revealing the subset of specific information about the study controlled for response bias.Therefore, the researcher introduced the study as research observing patient interactions with HCPs. If pushed further, the researcher would disclose that the research was focusing on various forms of educational interactions aimed at knowledge acquisition.Participants were informed that they could withdraw consent at any time for any reason without prejudice and participants could request that his or her data be totally discarded (Portney\& Watkins, 2009).

Immediate risk. The researcher sought and secured an acute hospital setting that was friendly, familiar, and in which it waseasy to establish rapport. The research setting includedHF patients and a health care team where the researcher connected with the team, mitigating risk or discomfort. This interconnectedness advanced a mutual understanding and accurate data interpretation (Marshall \&Rossman, 2011). However, conducting research in a familiar setting also posed threats of researcher bias, subjectivity, and inability to separate from the research, conditions the researcher was aware of and avoided by debriefing sessions, on the spot member checks, peer scruitiny of research study, and examination of previous research to frame findings (Shenton, 2004).

Personal consequences. The researcher had work experience with the research setting. Any concerns that arose during the study on the part of the HCPs or participants were addressed upfront and immediately. Any personal contact with participants at the conclusion of the study continued to be respectful so as to maintain and protect confidentiality of the data and edit reports in a manner that protects participant anonymity.

\section{Instrumentation}

Role of the researcher. In naturalistic research of a qualitative nature, the researcher is the instrument and mainstay for observation, data collection, and analysis (Lincoln \& Guba, 1985). As information becomes available, the researcher authenticated its efficacy and revised the analysisof the emerging data (Erlandson et al., 1993). The researcher created a mosaic of rich understanding and deep meaning from individuals or groups that supplied the data.

This researcher's previous experience as an auditor, educator, supervisor, case manager nurse, and social worker in diverse health care settings provided the foundation for conducting this research. These experiences shaped the researcher's proclivity to understand context, structure, and interactions involved within the hospital system for inpatient treatment of HF patients. Data collection primarily consisted of patient observations, a survey of expert providers, and content analysis for any education materials provided. Prior to data collection, the researcher practiced observations of patient and HCP exchanges in a simulated environment with two research faculty to test instruments, coding and analysis, and to ensure a proficient process.

Observation protocol. Well organized protocolsprovided a structured way for the researcher to record the patient provider interactions. In this study, an observation protocol was utilized to record patiet and HCP interactions (providers are physicians, nurse practitioners, nurses, case managers, social workers, dieticians, physical therapists, occupational therapists, speech therapists, chaplains). This protocol was derived from AHA HF protocols to ease the burden of data capture and allow for interpretation.

Field journal. This researcher kept an observational journal during time spent in the hospital setting, recording routines, and documenting the individuals tied to patient - provider interactions. This process advanced to noting ideas about the progression of the study in order to capture what was necessary for editing or reflection (Marshall \&Rossman, 2011; Richard \& Morse, 2007). A data collection strategy afforded noting observations 
on one side of the page, utilizing the second half of the page to narrate emerging analytic insights. The field journal helped the researcher debrief with peers and validate clusters, themes, or patterns in accumulating data.

Artifact collection. Artifacts can supplied background information that was used for justifying selection of the setting and enriching the analysis and interpretation of the research (Marshall \&Rossman, 2011). In this study demographic data obtained established that there were cardiology nursing divisions and HF patients. Focusing on other artifacts provided richness to the thick descriptions within the study, i.e., a HF campaign or health fair that includes HF patients and the health care team and patient education materials.

Demographic data sheet. Selected variables used to describe a sample were inherently quantitative (Polit \&Hungler 1999).Patient demographic datacollected at the start of the study are: age, gender, and race. Length of stay, ICU days, and secondary diagnoses were also collected.

\section{Sample}

The final sample consisted of $10 \mathrm{HF}$ patients in a 1000 bed Midwestern acute care hospital in the United States. Each HF patient was followed from the first day of admission through the day of discharge for a minimum of 10 hours of researcher time daily. The average length of stay for inpatient HF patients at this hospital wasseven days thus equating to 70 inpatient hospital days of observations. The sample of providers observed interacting with HF patients was $161 \mathrm{HCP}$ sall who consented to participate. These HCPs included physicians, nurses, nurse practitioners, dieticians, social workers, case managers, physical therapists, occupational therapists, speech therapists, and chaplains. The goal was to observe all potential sources responsive to meeting the educational needs of the HF patient.The primary researcher was the sole data collector. The study included English speaking patients between the ages of 50 to 80 years of age with a primary diagnosis of $\mathrm{HF}$ and NYHA classification rating stages of 3-4thatwas confirmed in the medical record. As a progressive disease, this purposive sample of patients had progressed to the point where patient education was a necessary intervention. Purposive sampling allowed the researcher to obtain a rich range of details specific to the study context. Specifically, this sample maximized the discovery of teaching - learning content, the source of information, and patient responses, allowing for patterns and themes to emerge. The plan was that if saturation or a point of redundancy was not reached with $10 \mathrm{HF}$ patients then 5 more HF patients would be added; this was not necessary as saturation was achieved with a sample size of 10.

Table 1identifies the cardiology floors by nursing divisions that were used to conduct this study.Table 3.1 Nursing Divisions Represented

\begin{tabular}{|c|c|c|c|}
\hline Nursing DivisionA & Nursing DivisionB & Nursing DivisionC & Nursing Division D \\
\hline $\begin{array}{l}\text { High risk cardiology; } \\
\text { admits with chest pain } \\
(\mathrm{CP}) \text {, arrhythmias, and } \\
\text { shortness of breath } \\
\text { (SOB) requiring } \\
\text { procedures and } \\
\text { intravenous cardiac } \\
\text { medicines/monitoring; } \\
\text { pre/post procedures or } \\
\text { medical/surgical admit } \\
\text { with cardiac risk }\end{array}$ & $\begin{array}{lr}\text { High risk } \\
\text { cardiology; admits } \\
\text { with } r \text { CP, } \\
\text { arrhythmias, and } \\
\text { SOB requiring } \\
\text { procedures and } \\
\text { intravenous cardiac } \\
\text { medicines/monitori } \\
\text { ng; pre/post } \\
\text { procedures or } \\
\text { medical/surgical } \\
\text { admit with cardiac } \\
\text { risk }\end{array}$ & 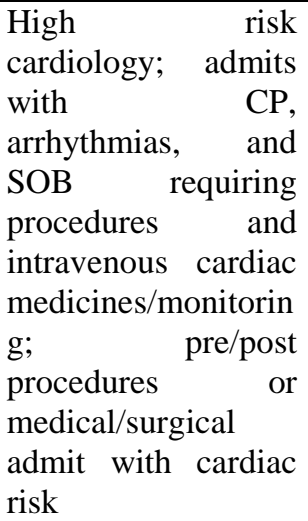 & $\begin{array}{l}\text { High risk cardiology; } \\
\text { admits with CP, } \\
\text { arrhythmias, and SOB } \\
\text { requiring procedures } \\
\text { and intravenous } \\
\text { cardiac } \\
\text { medicines/monitoring; } \\
\text { pre/post procedures or } \\
\text { medical/surgical admit } \\
\text { with cardiac risk }\end{array}$ \\
\hline
\end{tabular}

\section{Procedure for Data Collection}

Once the potential HF patients were identified from their admitting diagnosis and written participant consent was secured, the primary researcher observedHCP and HF patient interactions. Located towards the end of the hallway were open seating areas where the researcher waited untilHCPs entered the HF patient's room. Thishospital setting has an average length of stay of 7.0 days for HF patients. Given this 7.0 day length of stay timeframe theresearcher observedHCP and HF patient interactions according to plan with an alternating schedule of 7:00 am - 3:00 pm and 3:00 pm - 9:00 pm, based on the assumption that these time frames were interaction-rich and considering potential researcher fatigue. At the end of the field study, provider experts were surveyed according to the plan for five experts (internal medicine physicians, cardiologists, nurse practitioners) from each of the 4 nursing divisions. By transcribing HCP and HF patient interactions and reviewing field notes and artifacts, the researcher was fully immersed in the phenomenon of interest.

\section{Methods of Data Analysis}


Schatzman and Strauss (1973) characterized the process of data collection and data analysis as one unified function in order to build a coherent interpretation. Early in the research, the researcher seeks those experiences that lead to the development of understanding then moves towards the control of emerging ideas and then links these ideas into even more dense concepts and themes. In naturalistic inquiry, data analysis is open, inductive, and an interpretive process that leads to "maximal understanding of the phenomenon being studied in its context" (Lincoln \& Guba, 1985, p. 225). Data from this study included observations of HCP and HF patient interactions, analysis of educational content as evidenced through field notes and artifacts, a survey of expert providers (Appendix B), and demographic data (Appendix C).

The data was analyzed by the researcher and by a professional outside the context for peer debriefing which is a process that provides feedback for refining, redirection, and strengthens the analysis process (Erlandson et al., 1993). The following analytic procedures described by Marshall and Rossman (2011) were utilized: (1) organizing the data, (2) immersion in the data, (3) generating categories and themes, (4) coding the data, (5) offering interpretations through analytic memos, (6) searching for alternative understandings, and (7) writing the report. While raw data or "a rich description of ordinary events" (p. 210) had no inherent meaning, the process of data reduction and the interpretive act transformed data into findings.

Once observations of HCP and HF patient interactions through field notes were collected they were transcribed and coded (Erlandson et al., 1993). Then topics, regularities, and themes were coded so thatinformation could be retrieved by single categories or by a combination of categories. Viability of themes and explanations were compared against variations within the literature review and original conceptual framework to establish saturation of data (Marshall \&Rossman, 2011). As the researcher defined and established relationships between categories and themes, analysis emerged as an elegant, credible interpretation. The classification and integration of schemas during the analysis and interpretation process provided documentation for an external audit. In this study an experienced researcher outside the context was consulted to confirm coding methods and revise coding schemes for dependability and confirmability audits.

Earlier groundwork was examined to explore categories for subsequent analysis (Marshall \&Rossman, 2011). Likely themes suggested by the literature review were: systems of information, process of information, and management of information; patients with little understanding of the purpose of their medications; concerns about quantity and combination of drugs prescribed; difficulties in differentiating between the side effects of drugs and symptoms of HF; little knowledge regarding how to interpret and or treat changing symptoms of HF; prognosis and likely manner of death; successful communication with the doctor; difficulties in getting to hospital appointments; confusion andshort term memory loss; belief that doctors did not want to provide patients with too much knowledge regardingthe diagnostic process (identifying symptoms, seeking medical help, and coming to terms with the diagnosis); change in activities, life-style, work and self/identity; the role of others in adjusting to life with chronic illness; and negative and positive emotional reactions (Helleso, Eines, Sorensen, \&Fagermoen, 2009; Rogers et al., 2000, 2002; Thornhill et al., 2008). Well-organized data collection that is efficient and structured could use pre-existing categories; this was not done in order to discover salient themes and clusters from the data that existed within the study.

The data collected were compared and contrasted in an analysis of ideal HF education (AHA guidelines) versus actual $\mathrm{HF}$ education provided against what expert providers consider critical education needed for patients experiencing acute HF (Appendix D). The primary researcher examined congruency or differentiation between expected and actual HF patient education. Additionally, this researcher compared and contrasted what the provider believed as critical education for HF patient against HF education provided to the patient.

\section{Methods for Data Verification}

In naturalistic inquiry, older terms of reliability, validity, objectivity, and generalizability have been modified to include credibility, dependability, confirmability, and transferability in order to assure trustworthiness (Marshall \&Rossman, 2011). The seminal work of Lincoln and Guba (1985) has ensured that interpretations of ordinary language and complexities of individuals acting within a context are strong and credible as long as certain strategies and techniques are employed. The trustworthiness of this study was strengthened by following the strategies and techniques described below as credibility, transferability, dependability, and confirmability of data (Erlandson et al., 1993; Lincoln \& Guba, 1985).

Credibility. In naturalistic inquiry, the goal of credibility is to ensure that participants are properly identified and described and complexities of processes and interactions are accurately revealed. While threats to credibility cannot be ignored, such as the potential for findings to reflect the predilection or bias of the researcher, credibility/believability strategies and techniques require transparency. The study addressed credibility using the following techniques and strategies.

Prolonged engagement. Prolonged engagement estimates that the researcher spends enough time within the culture of the research setting in order to provide a foundation of credibility and reduce any distortions 
introduced by the newness factor such as researcher bias towards organization management style or teaching style. Furthermore, prolonged engagement builds rapport and trust with participants. This researcher has worked within this acute care setting for over 9 years,establishing the investigator's integrity and providing a solid background for trust and rapport. Going native, or becoming identified with the group being studied, was abetted with prolonged engagement.

Member checks. Member checks serve to provide credibility whereby the researcher verifies with the participant any themes, interpretations, and conclusions. The study included the following methods: summarizing data or rephrasing key ideas after an observed patient-provider exchange to allow for the immediate correction of errors; written opportunities to respond to themes and interpretations; and face to face opportunities to discuss themes as well. Participant feedback was integrated into the findings.

Triangulation. Triangulation established credibility whereby themes were confirmed by utilizing more than one source of data and more than one data collection method (Portney\& Watkins, 2009). This study followed the triangulation strategy by collecting data from more than one source and utilizingmore than one analytic approach.Furthermore, pieces of information (artifacts) were utilized to verify information secured through observed patient-provider exchanges, field notes, and observations.

Peer debriefing. Peer debriefing assisted in establishing credibility. This process allows for a disinterested peer to probe the researcher's biases, explore meaning, and clarify interpretations. The debriefer also discussed the research aims, pushed the researcher to test next steps within the emerging methodological plan, and provided opportunity for catharsis by helping to push the researcher towards the next steps. This study included a peer debriefer to review coding schemes, test research aims, and perform dependability and confirmability audits.

Reflexive journaling. Reflexive journaling was used to record notes on a regular basis reflecting on scheduling, insights, and reasons for methodological decisions (Erlandson et al., 1993). This process was done daily or entered weekly and added to credibility as the researcher's insights ultimately become a valuable piece of the audit trail.

Transferability. The naturalistic researcher maintained that the interrelationships and intricate details of a study could be transferred or applied to other contexts due to shared characteristics. The obligation of demonstrating transferability belongs to those researchers who apply it in a new or different context (Earlandson et al., 1993). Three strategies were used to facilitate transferability in this study: thick description, purposive sampling, and reflexive journaling.

Thick description. Thick descriptions provided sufficient detail of the data so that the reader could be brought into a particular well described context. Judgments or estimations about transferability can be made based on the similarities between sending and receiving contexts. Chapter Four provides detailed observations from the experience of knowledge acquisition for HF patients in an acute care setting.

Purposive sampling. Purposive sampling is similar to convenience sampling; however, there are specific requirements other than convenience. Naturalistic research seeks to maximize the discovery of patterns or problems that were provided rich details from a particular context. In doing so the researcher has no set rules for sample size. Thus, the use of purposive sampling enhances transferability. A purposive sampling was utilized to include all cardiology nursing divisions, excluding intensive care units,to seeksufficient information to fit the basic research questions and purpose of this study.

Reflexive journaling. Reflexive journaling enhances transferability through the rich details provided on the essence of the research setting. This study included transcribed notes on the hospital setting that were incorporated in Chapter Four.

Dependability. The naturalistic researcher utilizes dependability that correlates to reliability (stability, consistency, and predictability). By embracing stability and tracking instability, reality shifts, or design induced change, the naturalistic inquiry met the criterion of consistency. Consistency was realized as dependability in naturalistic inquiry and was revealed in a dependability audit. Two strategies were used to facilitate dependability: a dependability audit and reflexive journaling.

Dependability audit. A dependability audit increased dependability and consistency by conducting an audit that examined the processes used to conduct a study. An audit trail exposed documentation including raw data (observed patient-provider exchanges, field notes); data reduction and analysis products; data reconstruction; process notes; details on intentions; and, information related to instrument development (Erlandson, Harris, Skipper, \& Allen, 1993). A dependability audit was performed by the peer debriefer to confirm the purpose or aim of the study.

Reflexive journaling. A reflexive journal provided additional written information to support data collected coordinated with the purpose of the study.

Confirmability. The naturalistic researcher utilizedconfirmability that correlated with objectivity (findings relate to the focus of the inquiry not the biases of the researcher). Data was traced to their inception or origins and logic used for interpretations was authenticated explicitly and implicitly. Two strategies were used to expedite confirmability: a confirmability audit and reflexive journaling. 
Confirmability audit. The confirmability audit was similar to the dependability audit in that a sufficient trail was left for an external auditor to recognize whether the inquiry supported interpretations, conclusions, and recommendations and be able to trace their origins. In this study, the peer debriefer examined the inquiry context of the research.

Reflexive journaling. The reflexive journaling process was used to support the inquiry process. Any concerns that arise such as methodology issues were justified when conducting the study.

\section{Presentation and Dimension of Data}

An entire account of the findings from this study is found in Chapter Four. The research setting is disclosed, followed by a description of key themes and findings related to the research questions. The final results fully described the experience of how knowledge acquisition unfolds for HF patients in the acute care setting.

\section{Report of the Findings}

\section{RESULTS}

In this chapter, the findings are reported in two parts. Part A presents the demographics and the data collection process in the acute care setting where the research was conducted. Part B addresses the three research aims: (1) to determine what AHA Guidelines are utilized in the hospital setting; (2) to identify the HF instruction that went beyond the AHA Guidelines; and (3) to determine what experts felt was important instruction in the acute care stay. Observations beyond the research questions that provide contextual significance comprise the remainder of Chapter Four.

\section{Part A: Demographics and the Data Collection Process}

The research site and participants met the study criteria cited in Chapter Three. The acute care setting of a 1,000 bed academic teaching hospital located in the Midwest provided the opportunity to observe the interactions between providers of care and patients. The inpatient context was predetermined as a necessary factor to substantiate the presence of heart failure (HF) education at a vulnerable and important time in the patient's care trajectory. The final sample constituted $10 \mathrm{HF}$ patient participants who were selected based on their admission diagnosis of stage 3 or 4 congestive HF (chronic systolic and/or diastolic HF).

One hundred sixty one health care providers (HCPs) were observed during instructional interactions with the 10 patient participantsfrom April 30, 2014 through June 6, 2014,from day one of their hospital admission and each contiguous day thereafter through the entire length of stay. These interactions provided the focus for this research. HCPs and patients were aware from the informed consent that the study related to HF patient knowledge acquisition, i.e. instruction provided to patients and/or families, and was consistent with IRB feedback. HCPs were unaware that the researcher was specifically comparing and contrasting their teaching content to AHA Guidelines. The researcher was interested in the relevance of the AHA Guidelines designed for use in the acute care setting, whether instructional gaps existed, and the relevanceof who providedinstruction.

Instructional interactions between HCPs and HF patients occurred on four acute care cardiology units. An exception was made when a HF patient's acute care stay was interrupted by a transfer to the Cardiac Intensive Care Unit (C-ICU) at which time all observations were discontinued; there were three such transfers to the CICU. Observations of these patients resumed immediately once the patient was transferred back to one of the four acute care cardiac units. By design, all four cardiology units at the research site were included as part of this study because C-ICU patients are not always transferred back to the same room or acute cardiology unit due to census or other considerations. Tables 4.1 and 4.2 present detailed demographic information on the $10 \mathrm{HF}$ patients and the $69 \mathrm{HCPs}$, respectively.

Table 4.1 HF Patient Demographics (n=10)

\begin{tabular}{|c|c|c|c|c|c|c|c|c|}
\hline Patient & $\begin{array}{l}\text { Primary } \\
\text { Diagnosis }\end{array}$ & $\begin{array}{l}\text { Secondary } \\
\text { Diagnosis }\end{array}$ & Age & Gender & Race & $\begin{array}{l}\operatorname{LOS}^{1}, \\
\mathrm{ICU}^{2} \\
\text { (days) }\end{array}$ & Unit(s) & $\begin{array}{l}\text { Admit } \\
\mathrm{DC}^{3} \\
\text { dates }\end{array}$ \\
\hline$\overline{\mathrm{A}}$ & $\begin{array}{l}\text { Heart } \\
\text { Failure } \\
(\mathrm{HF})\end{array}$ & $\begin{array}{l}\text { Coronary } \\
\text { Heart } \\
\text { Disease }\end{array}$ & 64 & Male & "Caucasian & 10,1 & B \& D & $\begin{array}{c}4 / 30 / 14 \\
-5 / 9 / 14\end{array}$ \\
\hline B & $\mathrm{HF}$ & $\begin{array}{l}\text { Pulmonary } \\
\text { Hypertensio } \\
n\end{array}$ & 56 & Female & $\begin{array}{l}\text { African } \\
\text { American }\end{array}$ & 6 & A & $\begin{array}{r}5 / 2 / 14 \\
-5 / 7 / 14\end{array}$ \\
\hline $\mathrm{C}$ & $\mathrm{HF}$ & Acute Renal & 78 & Female & Caucasian & 3 & $\mathrm{D}$ & $5 / 13 / 14$ \\
\hline
\end{tabular}




\begin{tabular}{|c|c|c|c|c|c|c|c|c|}
\hline D & $\mathrm{HF}$ & $\begin{array}{l}\text { Failure } \\
\text { Pneumonia }\end{array}$ & 52 & Male & Caucasian & 11,2 & A & $\begin{array}{r}-5 / 15 / 14 \\
5 / 13 / 14 \\
-5 / 23 / 14\end{array}$ \\
\hline E & $\mathrm{HF}$ & $\begin{array}{l}\text { Acute } \\
\text { Bronchitis }\end{array}$ & 55 & Female & $\begin{array}{l}\text { African } \\
\text { American }\end{array}$ & 3 & B & $\begin{array}{r}5 / 16 / 14 \\
-5 / 18 / 14\end{array}$ \\
\hline $\mathrm{F}$ & $\mathrm{HF}$ & $\begin{array}{l}\text { Diabetes } \\
\text { Mellitus } \\
\text { Type II }\end{array}$ & 75 & Female & Caucasian & 7 & B & $\begin{array}{c}5 / 19 / 14 \\
-5 / 25 / 14\end{array}$ \\
\hline G & $\mathrm{HF}$ & $\begin{array}{l}\text { Atrial } \\
\text { Fibrillation }\end{array}$ & 54 & Male & Caucasian & 4 & $A \& D^{3}$ & $\begin{array}{r}5 / 25 / 14 \\
-5 / 28 / 14\end{array}$ \\
\hline $\mathrm{H}$ & $\mathrm{HF}$ & $\begin{array}{l}\text { Central } \\
\text { Retinal } \\
\text { Artery } \\
\text { Occlusion } \\
\text { w/ } \\
\text { Resolution }\end{array}$ & 52 & Male & $\begin{array}{l}\text { African } \\
\text { American }\end{array}$ & 7 & $B \& C^{4}$ & $\begin{array}{r}5 / 28 / 14 \\
-6 / 3 / 14\end{array}$ \\
\hline I & $\mathrm{HF}$ & $\begin{array}{l}\text { Atrial } \\
\text { Flutter }\end{array}$ & 66 & Male & Caucasian & 7,2 & A \& D & $\begin{array}{c}5 / 29 / 14 \\
-6 / 4 / 14\end{array}$ \\
\hline $\mathrm{J}$ & $\mathrm{HF}$ & $\begin{array}{l}\text { Cocaine } \\
\text { Related } \\
\text { Chest Pain } \\
\text { w/o } \\
\text { Myocardial } \\
\text { Infarction }\end{array}$ & 60 & Male & $\begin{array}{l}\text { African } \\
\text { American }\end{array}$ & 1 & $\mathrm{~B}$ & $\begin{array}{r}6 / 6 / 14 \\
-6 / 6 / 14\end{array}$ \\
\hline
\end{tabular}

Note. ${ }^{1}$ Length of stay. ${ }^{2}$ Intensive care unit. ${ }^{3}$ Unit change due to leak in ceiling. ${ }^{4}$ Unit change due to request for private room.

Table 4.2 HCP Demographics (Clinical and *Non-clinical) Who Provided HF Education ([HFE]; n=69)

\begin{tabular}{|c|c|c|c|c|c|c|c|c|}
\hline HCPs & $\begin{array}{l}\# \text { HCPs } \\
\text { providing } \\
\text { HFE }\end{array}$ & $\begin{array}{l}\% \text { HCPs } \\
\text { providing } \\
\text { HFE }\end{array}$ & $\begin{array}{l}\text { Mean } \\
\text { (SD)Age } \\
\text { Range }\end{array}$ & F N & & AA & & \\
\hline RNs & 23 & 56 & $\begin{array}{l}42(10.88) \\
24-63\end{array}$ & 23 & & 5 & 1 & 17 \\
\hline MDs & 23 & 92 & $\begin{array}{l}38(20.73) \\
26-74\end{array}$ & 4 & 19 & 7 & 16 & \\
\hline NPs & 7 & 100 & $\begin{array}{l}39(7.16) \\
30-53\end{array}$ & 7 & & 7 & & \\
\hline FS & 6 & 50 & $\begin{array}{l}33(15.53) \\
20-60\end{array}$ & 3 & & 6 & & \\
\hline RTs & 3 & 27 & $\begin{array}{l}46(12.98) \\
24-61\end{array}$ & 2 & 1 & 3 & & \\
\hline SWs & 3 & 100 & $\begin{array}{l}46(10.41) \\
38-58\end{array}$ & 2 & 1 & 3 & & \\
\hline RDs & 2 & 100 & $\begin{array}{l}25(2.12) \\
23-26\end{array}$ & 2 & & 2 & & \\
\hline PCT & 1 & 1 & $\begin{array}{l}31(13.48) \\
22-61\end{array}$ & 1 & & 1 & & \\
\hline $\mathrm{HK}^{*}$ & 1 & 20 & $\begin{array}{l}45(9.75) \\
33-57\end{array}$ & 1 & & 1 & & \\
\hline
\end{tabular}

Note. *Non-clinical. HCPs ( $\mathrm{n}=39)$ who did not provide HFE are not shown in Table 4.2: case managers, physical therapists, occupational therapists, nurse educator, patient access worker, chaplain, heart and vascular concierges, transporters, and secretaries. $\mathrm{M}=$ Male; $\mathrm{F}=$ Female; $\mathrm{AA}=$ African American; $\mathrm{A}=\mathrm{Asian} ; \mathrm{C}=$ Caucasian.

Demographics. A total of $10 \mathrm{HF}$ patients were observed, which included six males and four females ranging from 52 to 78 years of age, with a mean $(\mu)$ age of $61( \pm 9.38)$. Six HF patients were Caucasian and four patients were African American. Average length of stay was 6.4 days $( \pm 3.18)$.In addition to the primary diagnosis of HF, each patient had secondary diagnoses, including acute bronchitis, acute renal failure, atrial fibrillation, atrial 
flutter, central retinal artery occlusion with resolution,cocaine related chest pain, Diabetes Mellitus type II, pneumonia, and pulmonary hypertension. These secondary diagnoses alerted the researcher to observe HF education that was both based on defined standards as well as based on comorbidities. Of the 161 HCPs observed during the study, $69(43 \%)$ provided some form of HF education. Those HCPs who provided HF education included: nurses $(n=41)$, physicians $(n=25)$, patient care technicians $(n=16)$, food service workers $(n=12)$, respiratory therapists $(n=11)$, nurse practitioners $(n=7)$, housekeepers $(n=5)$, social workers $(n=3)$, and registered dieticians $(n=2)$. Note that one non-clinical worker, a housekeeper, provided instruction to a patient, a role not typically aligned with patient education. The following HCPs interacted with patients and could have provided instruction yet were not observed doing so: case managers $(n=5)$, physical therapists $(n=5)$, occupational therapists $(n=4)$, a nurse educator $(n=1)$, and a chaplain $(n=1)$. Non-clinician HCPs who interacted with patients also did not provide HF education: transporters $(n=15)$, heart and vascular concierges $(n=3)$, secretaries $(n=4)$, and a patient access worker $(n=1)$.

The blend of racial diversity between HCPs (Caucasian $n=48,63 \%$; non-Caucasian, $n=21,37 \%$ ) and HF patients (Caucasian, $n=6,60 \%$; non-Caucasian, $n=4,40 \%$ ) was relatively well matched. Some studies suggest race concordance is associated with patient satisfaction and/or patient outcomes. The fact that a close blend of matched diversity was an important factor in mitigating diversity is a special issue for analysis in this study (Albert et al., 2009; Blustein et al., 2008; Street et al., 2008).

Data Collection Process. A total of 31,920 minutes (532 hours) was spent on the clinical units, preparing for the observations (i.e., chart review), waiting for HCPs to visit the patient, and ultimately observing the HCPs when the patient was visited. HF education comprised 911.1 minutes (15.19 hours) in actual instructional interactions between HCPs and patients. These 15.19 hours of data form the basis for analysis in response to the research questions. HF educational interactions ranged from 30 seconds to 50 minutes.Multiple teaching resources (artifacts) were coded to augment the observational findings.

The researcher utilized prolonged engagement, persistent observation, triangulation, peer debriefing, and on the spot member checks to ensure credibility (internal validity). In naturalistic inquiry, the description developed by the researcher must ring true for the person of that setting. One patient jokingly commented, "there's the hawk [research nurse sitting on a chair in the hallway]." Some HCP's comments were, "Are you doing ok?" and "Are you getting what you need?" These comments connoted a welcoming attitude to the researcher's presence. The protocol documentation sheet that was developed permitted ease of effort in documenting time, content, and responses to instructional initiatives. With each encounter, reflexive journal entries (field notes) were written for review and summarization at the end of each day before transcription into Microsoft word. Artifacts that encompassed instructional HF content (brochures found and used within the cardiology unit including the patient's room, the nurse's station, the waiting room, and hospital lobby) were collected and coded separately from verbal interactions.

Member checks, audit trails, and peer debriefings were performed to ensure the credibility of research findings. As needed, the researcher verified or clarified content and later interpretations, themes, or conclusions with HCPs and patients to offer them an opportunity to respond to or correct interpretations. A peer faculty debriefer helped to establish a proficient observation technique for patient-provider exchanges in a simulated environment. One other faculty tested instruments, received coding, and analysis to confirm that both data and interpretations were verifiable. Further the peer debriefing explored any prevailing biases, meanings, and interpretations. The debriefer challenged the researcher to suspend identifying themes too early in the data collection to avoid clouding judgment. The debriefer also randomly selected items from the protocol to verify that coding schemes and categories aligned, thus assuring a dependability and confirmability audit. These audits authenticated consistent findings and showed agreement with the organization of the data. The observation protocol, reflexive journaling, and artifacts were evaluated as part of the audit trail.

The data was organized by the research aims: (1) HF education related to the AHA Guidelines, (2) HF instruction beyond the AHA Guidelines, and (3) survey responses provided by expert HCPs. These findings are reported in the next section. Utilizing a process of descriptive content analysis allowed for patterns and themes to emerge. These patterns and themes are alsodetailed next.

\section{Part B: Three Main Research Aims and Questions}

Data analysis for the research questions are presented in this section. The first research aimcompared actual data collected using AHA'sGet with the Guidelines-Heart Failure Enhanced Heart Failure Patient Education Prior to Hospital Discharge Guidelines as the analytic framework (hereinafter referenced as the AHA Guidelines). The second question discerned instructional topics covered by HCPs but not identified in the AHA Guidelines. The third question revealed those topics an expert panel determined as essential to cover in a rapidly changing and demanding clinical environment.

Analysis of the data linked to the first two research questions revealed that nearly the same amount of time was spent covering topics tied to AHA evidence-based topics as the one non-AHA topic that was 
discovered, showing $10 \%$ variation in time ( $\mathrm{AHA}=498.6$ cumulative minutes and non- $\mathrm{AHA}=412.5$ minutes). This suggested early on in the analysis that a dynamic existed in recommended evidence-based instruction and clinical realities. This one non-AHA instructional topic was unique to the acute hospital stay yet was missing as part of AHA expectations for HCP instruction necessary for improved patient outcomes.

Research Aim 1 (RA1): Instructional content aligned with AHA guidelines. For research aim 1,the research questions were: (a)How much total time was spent on AHA topic related instruction? (b) Which AHA topics and content were covered? (c) How much time was spent on each AHA topic related instruction? (d) Which HCPs provided the instruction for each AHA topic? The data collected and coded by AHA topic and sub-topics (explained below) revealed that $498.6( \pm 67.75)$ minutesof $\mathrm{HF}$ instruction was spent between the 10 patients studied. The standard deviation and the range (0.5 minutes to 259.7 minutes) showed dramatic variability between patients.

Appendix F contains the complete set of AHA guidelines. There are nine primary topical areas of focus identified in AHA Guidelines, and all but the last three have sub-topics. In Table 4.3 data are organized in rank order of time spent in instruction, with primary areas of focus presented in bold. Each primary area is subsequently discussed in detail.

Table 4.3AHA HF Topics byNumber, Time, andHCPs $(n=69)$

$\mathrm{HF}$

Topics Total

Addressed Time

w/ Patients perTopi HCPs Providing

Number c HF Education

AHA HF Topics

(\%)

(min.) (min.)

Medicines/Type \& Use*

$10(100)$

Low Sodium Diet*

Follow-up Appointments*

Exercise*

Discharge Instructions

Topics)*

Daily Weights*

Symptom Recognition*:

Fluid Buildup ${ }^{1}$

Shortness of Breath ${ }^{1}$

Actions to Take for Weight ${ }^{2}$

Increase (Fluid Overload) ${ }^{2}$

Eat Healthy $^{3}$

Risk Modification*:

NoSmoking ${ }^{3}$

End of Life*

Cough $^{1}$

Tired/Fatigue ${ }^{1}$

Changes in Body Weight ${ }^{1}$

Increased Heart Rate ${ }^{1}$

Lack of Appetite ${ }^{1}$

Compare weight to dry weight ${ }^{2}$

Normal/Abnormal Weight Gain ${ }^{2}$

Maintain Target Blood Pressure ${ }^{3}$

Maintain Weight ${ }^{3}$

Exercise Expected Changes ${ }^{4}$

Warm Up Exercises ${ }^{4}$

How Long to Exercise ${ }^{4}$

Cool Down Exercises ${ }^{4}$

Limit Alcohol $^{5}$

$1(10)$

$5(50)$

1(10)

$1(10)$

$1(10)$

1(10)

0

0

0

0

0

0
259.7

$\mathrm{MD}=1-3 \quad \mathrm{NP}=4$

$$
\mathrm{RN}=1-19 \quad \mathrm{RT}=3
$$

$\mathrm{FS}=1-10 \mathrm{RD}=2 \mathrm{MD}=1-1.7$

$68.95 \quad \mathrm{PCT}=1 \mathrm{RN}=1.5-5 \mathrm{SW}=10$

44.7 $\mathrm{MD}=1-1.7 \mathrm{NP}=2 \mathrm{RN}=1-5$

$43 \quad \mathrm{RN}=1-2 \quad \mathrm{HK}=40$

$\begin{array}{ll}8(80) & 43\end{array}$

$25 \quad \mathrm{RN}=25$

16.25

$\mathrm{MD}=0.5-5 \mathrm{RN}=1-2.75$

15.5

$\mathrm{MD}=2-2.5 \mathrm{RN}=1-4$

14.75

$\mathrm{MD}=0.5-5 \mathrm{RN}=1-2.75$

$\mathrm{RN}=3.75$

$\mathrm{RN}=2$

$\mathrm{RD}=3$

$\mathrm{MD}=1$

$\mathrm{MD}=0.5$

$\mathrm{MD}=0.5$ 
Literacy Assessment ${ }^{6}$ 0

Plan for Refilling Prescriptions ${ }^{6} \quad 0$

Note. *AHA Guidelines Major Topics (Discharge Instructions include: symptom recognition, exercise, medications, daily weight, diet, and follow-up). ${ }^{1}$ Sub-topic of symptom recognition. ${ }^{2}$ Sub-topic of daily weights. ${ }^{3}$ Sub-topic of risk modification. ${ }^{4}$ Sub-topic of exercise. ${ }^{5}$ Sub-topic of low sodium diet. ${ }^{6}$ Sub-topic of medications.

Figure 4.1. AHA HF Instruction by Topic and Minutes

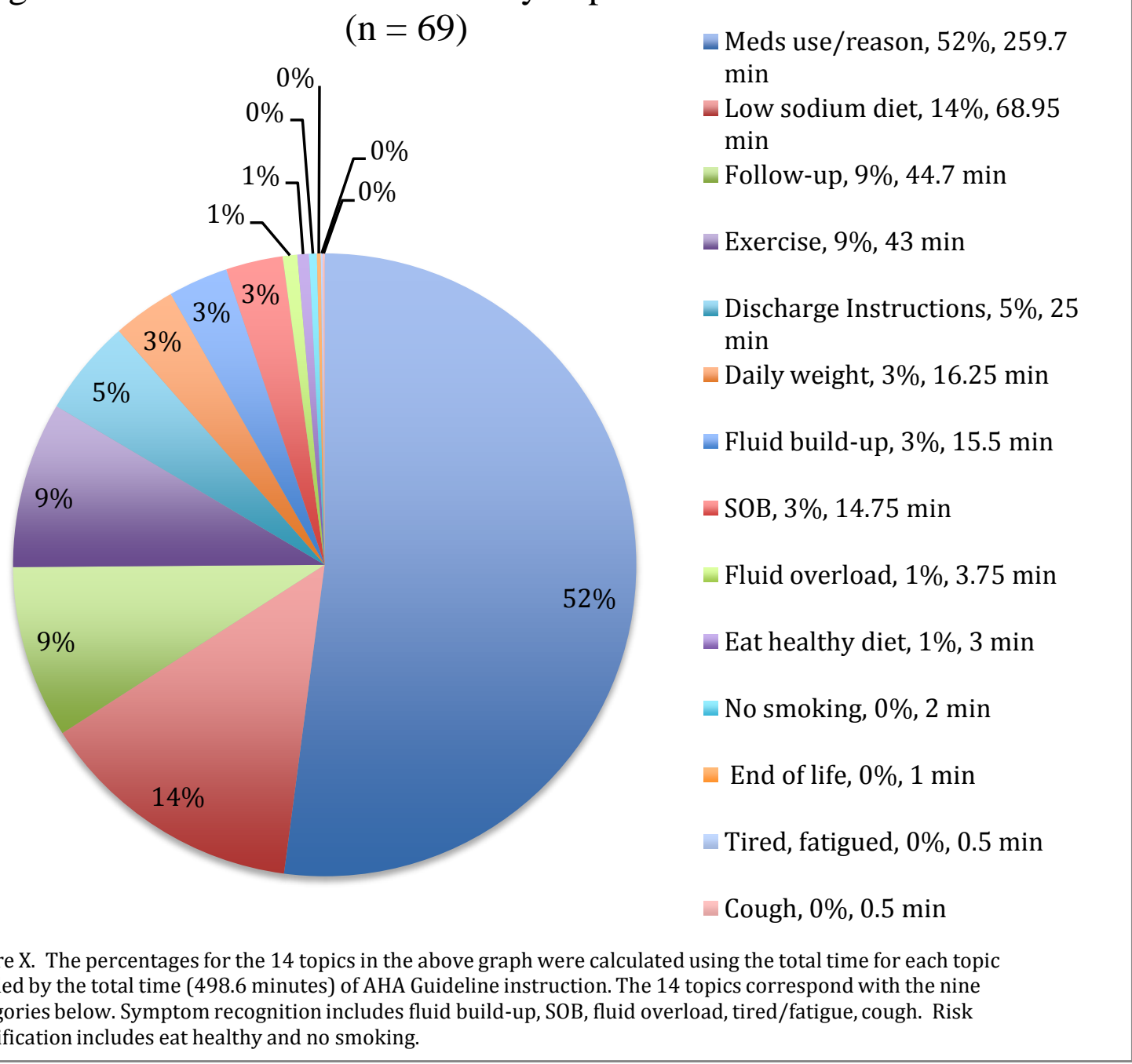

Category 1: Medications. Medication is one of the dominant therapies for HF, yet poses high risk for life threatening side effects that could trigger hospitalization. Ensuring that patients adhere to medication regimensis linked to improved health outcomes (Baker et al., 2011; Driscoll, Davidson, Clark, Huang, \& Aho, 2009). AHA Guidelines recommend instruction in the name, use, and need for medication, expected side effects, handling a missed dose, and planning for refills. The AHA Guidelines include a standard that a literacy assessment be conducted so as to match the patient's literacy level with the instructional level (Beckelman et al., 2011; Rosland, 2011).

All 10 patients received some form of instruction on medications, totaling 259.7 minutes of instructional effort. The time range spent per patient was broad, from 1 to 19 minutes $(M=5.7 ; S D=4.4)$, with nurses and physicians providing most of the instructional time. Nurses administer medications, giving them the greatest opportunity to instruct patients concurrent with administration. Physicians, a nurse practitioner, and a respiratory therapist also provided content instruction. A pharmacist might play a role in medication instruction, given their expertise. In this setting the role of the pharmacist was observed to be limited to medication delivery. Two topical areas HCPs failed to include were in patient literacy and post-hospital plans for refilling prescriptions. At no time during 532 hours of observation did the researcher observe an assessment of patient 
literacy. Unlike other standards, this guideline stands out because it is not an instructional topic, but a provider assessment that can be linked to other topical areas beyondteaching about medications.

Three examples show the range of instruction observed: timing of medication administration, linking medication with heart function, and refilling prescriptions. In the first, the patient had a history of medication noncompliance, but stated readiness to change this based on disease progression. Wanting the nurse to provide a medication list and timing schedule, the nurse asked, "What time do you start your day?" She tailored a patient-specific administration schedule and verified understanding all within six minutes. This example links together the importance of a patient's readiness to learn, customizing a standard in an adaptable manner and knowledge validation; all accomplished within a focused teaching session. In another example, a physician cupped his hands together to represent the heart as he instructed a patient on how a medication would impact ejection fraction. Describing ejection fractions and stroke volumes and mathematical formulas in an eight minute time frame was observed with no patient response or assessment of the patient's health literacy level. The well-intended effort was documented and could have been useful had knowledge validation occurred. The final example models the complexity of HCPs providing instruction for the post-hospital setting, specifically aimed at refilling medications. It became clear that the location of pharmacies, insurance coverage, and other access and physical activity factors are variables unknown to HCPs in the acute care setting, reducing their instructional ability to provide meaningful patient instruction. This reality was observed in a patient who was discouraged by his physician specialist from using the inpatient pharmacy service offered to inpatients at discharge to fill prescriptions, yet this would have provided the patient with a 30 day supply of medicines and waived copays. The physician directed the patient to contact his outpatient doctor if he is unable to pay for prescriptions. The patient noted that his discharge planning form stated, "Talk with your [primary care] doctor if paying for your medicines is a problem." The confusing nature of information and what could have been a quick fix 30 day supply of medications provided at dismissal created the likelihood that this patient would either return in 30 days for a refill, or a scenario where the patient conceivably would go without. Hospital penalties for readmission do not account for a patient's social circumstances (AHA, 2011).

Category 2: Diet. A low sodium diet is a way to avoid fluid retention, a caustic issue for the HF patient. HCPs play a role in instruction that addresses the problems caused by sodium intake, identifieshigh sodium foods, and deals with patient complaints that sodium-restricted foods often lack appeal. AHA Guidelines support the dangers of fluid retention in exacerbating heart failure without sodium monitoring and compliance (Grady et al., 2000). Diet instruction includes both solids and liquids. Diuretic medications (such as Lasix) often create thirst, and fluid restrictions can add to a patient's desire for fluids (Baker et al., 2001; Driscoll et al., 2009). Instructional content on flavoring food without sodium and mitigating thirst through alternatives such as ice chips or hard candies, serves a clinical purpose to minimize fluid intake, and provides comfort.

Eight of the 10 patients received instruction on low sodium diets with the amount of time spent on this topic ranging from one to 10 minutes $(\mathrm{M}=2.9 ; \mathrm{SD}=2.6)$. Food service workers (non-dieticians) and nurses provided dietary education to nine patients, supported by physicians (who instructed three patients), and a dietician, a PCT, and a social worker who each reached out to one patient.Diet instruction was interspersed throughout the patient stay. Considering the role of the dietician as a nutrition expert, diet instruction was not consistently observed as part of this role.

The AHA Guidelines also call for dietary instruction on alcohol restriction or abstinence which Abramson, Williams, and Krumholz (2001) cite as heart toxic. While alcohol intake was a negligible issue for these patients while hospitalized, the topic may have had relevance post-discharge.

In the cases observed where diet instruction was provided, one pattern was noted - patients find food and fluid restrictions difficult to bear. Some patients asked for specific foods, only to be dissuaded from that choice, often with sensitivity and even humor. HCP instructions acknowledged food and fluid sacrifices and efforts were made to help patients cope with these difficult changes. Simple comments kiddingly made like, "Good news, the tartar sauce was approved by the nurse [for your fish]" helped ease the burden. One physician provider was very direct and instructed a patient to ensure low sodium foods "because your heart doesn't have a lot of reserve" to accommodate high sodium foods. One final example of instruction was when a nurse provided yet another patient with a fluid measurement chart and strategies for choosing comparable foods with less liquid intake. Of the instructional categories observed, the area of diet instruction was most patient-centered.

Category 3: Follow-up appointments. HF requires constant treatment, not justin the hospital setting. Follow up care is essential for ongoing clinical management. It should be evident from the aforementioned categories of instruction and data that follow up care may be as or more important than hospital-based care. Patients require frequent contact with their HCPs for treatment adjustment which can sustain comfort and avoid hospitalization. AHA guidelines recommend that HCPs provide patients with rationale for follow up appointments, and at the time of dismissal ensure that follow up appointments are written with the date, time, and location of follow up care. 
Eight patients received a total of 44.7 minutes of instruction on follow up appointments, ranging from one to five minutes $(\mathrm{M}=2.5 ; \mathrm{SD}=1.3)$. Physicians and nurses werethe dominant educators. Follow up instructions were interspersed throughout the stay dispelling notions that follow up care is rushed at the time of discharge. Several examples are noted: physicians were central in connecting patients with outpatient HCPs, nurses reviewed follow up appointments with patients in writing, as the guidelines recommended, and other HCPs (nurse practitioners and social workers) were instructive to patients. A social worker from the Stay Healthy Outpatient Program (SHOP) offered free follow up services to a patient for up to two months for outpatient resource assistance. This service was designed to impact heart failure readmissions. Manning (2011) underscored that intensive one-to-one teaching of HF patients during hospitalization must be tied to follow up care, improved self-care management, quality of life, reduced readmissions, and societal costs.

The hospital requires that each patient have documented discharge instructions on a form designed to provide critical follow-up information. An audit of this form revealed that seven patients had incomplete documentation in the specific area of follow up care which was to include the name of the practitioner, location, and date/time of appointment. This complete information was provided to three patients. Fleming \& Haney (2013) reference follow up instructions as closing the gap between hospital discharge and first outpatient visit with a HCP.

Two illustrations model the confusion caused to the patient when follow up information is incomplete. One HCP explained to the patient the need to see both her heart doctor and general medicine practitioner. Discrepancies on what was said and what was written on the discharge paperwork were noted, such as the time frame to see the heart specialist $(2-4$ weeks instead of the $1-2$ weeks verbalized). No general medicine physician appointment was established and/or documented. Another physician stated he would write out instructions for follow up appointments on the discharge form. What was observed was that a phone number was recorded along with instruction for the patient to contact his (primary care) doctor for an appointment. In general, analysis reflectedthat follow up appointments were frequently omitted on discharge forms.

Category 4: Activity and exercise. As a muscle, the heart needs exercise, but when damaged, this complicates things. The AHA Guidelines activity and exercise category presents opportunities to prescribe exercise and how too little or too much can create negative clinical sequelae (Keteyian, 2011).

In this category, three patients received instruction, with a sparse 43 minutesspent between them. The mean time spent on this topic was 14.3 minutes $(\mathrm{SD}=22.23)$, ranging from one minute to 40 minutes. Exercise was rarely discussed, yet these patients had deconditioning and physical limitations linked to their stage 3 or 4 HF. One patient was assessed by PT and OT without instruction on conditioning or moderate exercise from bed to chair.

For the three patients receiving exercise instruction, exercise warm-up, cool-down, expected physiologic changes, and length of exercise was not covered. Another patient was ordered to exercise for 20 minutes on a treadmill twice while hospitalized. No HCP provided instruction before or after the exercise period. No instruction about the different kinds and length of exercise, frequency, expected physiologic changes, or warm-up and cool-down regimens was offered, all important heart rate recovery predictors for mortality noted by Yilmaz and coworkers (2013).

A single patient received 40 of the 43 minutes of the instruction provided. The instruction was initiated when the patient mentioned to a housekeeper about "not being able to walk like in the past." The housekeeper conversationally began to talk about an exercise regimen that included how to pace oneself when walking around the block, how to consider weather elements as an exercise factor, and more. The conversation progressed in a relaxed manner and with an open agenda that included how to stay motivated and where to gain more knowledge about exercise; the information shared was accurate. This conversation had the potential to increase self-management knowledge and delay disease progressions, consistent with the findings of Roncalli and colleagues (2009). The patient verbalized a plan to increase activity as a result of the interaction with this non-clinical worker.

Category5: Discharge instructions. Discharge instructions should be a culmination of patient education provided throughout the hospital stay. Validating treatment and reinforcing the patient's ability to recognize symptoms, evidence shows, improves self-care management and is fundamental to learning (Bandura, 1986; Guskey, 1985; Lovenmark et al., 2012). Furthermore, support provided at discharge has shown to reduce allcause mortality, length of stay, and improved quality of life (Phillips et al., 2004). As noted above, a discharge form was designed to provide written instructions to patients with HF. The design of the form accommodates some of the AHA Guidelines, but migrates between clinical information useful to post-hospital HCPs, and lacking congruency with the HF experience of patients. For instance, the form lists allergies, pneumonia and influenza screening, and other peripheral information useful to post-hospital HCPs, and does accommodate symptom, diet, weight monitoring, medications, activity, and follow up appointments. There is not a place for documentation to occur in the health record beyond this form. 
One patient received discharge instruction from a nurse (this category includes six categories: symptom recognition, exercise, medication, daily weights, diet, and follow up appointments). The total time spent on this topic was 25 minutes. The patient also had AHA teaching on three categories during her stay that were observed being reinforced during discharge instructions. Nurses are in a unique position because of their close and frequent encounters with patients to ensure patients understand key aspects of post-hospitalization care. Making learning (i.e., the six categories of discharge instructions) into an activity during provider-patient exchanges and intertwined with other clinical routines is one way to promote patient recall, mastery, and selfefficacy.

As designed, six of the topical categories prompt the HCP to instruct and document instructions. Nine patients had some kind of documented discharge instructions provided. Five of the nine received instruction in two to four categories of the AHA Guidelines; one patient had five categories covered. The following illustration shows that instruction at discharge was incomplete. One patient received discharge instructions from a nurse who was rapidly reading through the discharge paperwork. The patient stopped the nurse on the medication section for more focused teaching that addressed the patient's most pressing concerns. Instruction on daily weight was missed due to the arrival of the patient's ride home. Instruction on risk modification (e.g., smoking cessation) and end of life education was also not included and not appropriate for this patient.

Discharge instruction is a process that can cumulatively evolve so that by dismissal, reinforcement of instruction is achieved. One patient had stayed at a rehabilitation facility prior to hospitalization and now was planning to return home at discharge. During her hospital stay, the team provided the patient instruction on medication, follow up appointments, and diet. Reinforced at discharge were daily weights, symptom recognition, and exercise. A review of these important instructions acknowledged with the patient and a family member that the patient would have to adapt to a new point in the trajectory of this condition and resume self-care at an appropriate/negotiated level of engagement (Chen et al., 2011).

Category 6: Daily weights. The AHA Guidelines acknowledge that taking a daily weight is a critical patient activity. Patient vigilance to taketheir weight and act on weight gains is crucial, because weight is an early predictor of other more dire symptoms such as increased heart rate, shortness of breath, and swelling throughout the body which, together, could require hospitalization. AHA guidelines recommend patient instruction that the daily weight be compared to a "dry" weight. The literature varies on how to determine a dry weight but for HF patients it is likely the weight taken as the patient awakens, when blood pressure is in a normalized range, and visual edema is absent. Once recorded, this weight becomes the standard by which the daily weights are compared to determine if fluid overload is present and linked to heart function. These specifics are presented because it is an example of how much is demanded of HCPs in instructing patients in areas that lack precision, but require finely honed clinical judgment (Zhu \& Levin, 2011). It also demonstrates the clinical knowledge that HF patients and their families must possess to manage the disease.

A nurse or physician instructed five out of 10 patients to record a daily weight. In total, 20 minutes was spent on weight-related topics, with a range of 0.5 to 5 minutes $(M=2.2 ; S D=1.43)$. Daily weight is rarely discussed at admission, but does get introduced near the halfway point in the stay and again toward dismissal. Although nurses and patient care technicians took a daily weight of each of the 10 patients, this activity was not accompanied by instruction.

HCPs were not observed helping the patient determine a dry weight, nor was instruction given on calculating the difference between weights to determine and act on risk for HF overload. The issue associated with determining dry weight may contribute to topic avoidance. Further, the patient's math skills would need validation to ensure validity and reliability of reporting results to HCPs.

As the research progressed and data were recorded, it became clear that the topics noted in the AHA guidelines that appear mutually exclusive are not. Weight monitoring is a topic nested in other categories, including symptom management and risk modification.

Two exemplars typify the instruction given by HCPs related to weight, and both occurred at discharge. The first patient was instructed to take a daily weight, with no mention of documenting the weight, or determination if the patient had a scale. The second patient was instructed on the importance of weight monitoring and record keeping. Admitted to the hospital with a HF-related 30 pound weight gain, developing a dry weight baseline and reinforcing the weight-HF link would seem to be an important topic for instruction, a missed opportunity.

Category 7: Symptom recognition. As noted in Chapter Two, the importance of educating patients on symptom recognition is critically important and it is not surprising that this topic is central to the AHA Guidelines. The timeliness of recognition of and acting on symptoms (including contacting a provider) may determine survival. There are key sub-categories of symptoms that AHA guidelines recommend be covered: shortness of breath, cough, fluid build-up, being tired, or showing fatigue, experiencing lack of appetite, showing increased heart rate, and monitoring body weight. As is evident, this category is comprehensive and 
would require focused instructional efforts by HCPs in order to achievethoroughly educating patients in recognizing symptoms.

In this category, seven of the 10 patients received education, provided exclusively by either a physician or nurse. Altogether, 31.25 minutes was observed covering the topicand sub-topics in this entire category, ranging from 0.5 minutes to 5 minutes; $(\mathrm{M}=4.46$; $\mathrm{SD}=1.3)$ per patient. Keokkoek and colleagues $(2011)$ advise that instruction could improve patient self-management, reduce risk for rapid decompensation, and reduce admissions.

Not all of the sub-topics were covered in observed instruction. While four areas were covered: shortness of breath, cough, fluid buildup, and tiredness/fatigue, missing was the focus on lack of appetite and increased heart rate. There may be factors that undergird why these topics were not covered. According to Chappa and coworkers (2014) and Krumholz and colleagues (2000), lack of appetite may signal other problems beyond heart failure, such as depression resulting from the illness. Similarly, increased heart rate may be difficult to assess, as heart rates are normally variable based on activity, the effects of prescribed medications, and other factors. A possible explanation is that teaching about heart rate as a symptom may have too many decision points to teach and reflect a level of nuanced clinical judgment beyond the patient's capacity. Still, Desai (2012) and Fallis and colleagues (2013) indicate that heart rate changes tie to early detection, reporting, and promptness for treatments which may be lifesaving.

Clinical exemplars of the above are noted. A nurse asked one patient about fluid buildup to verify symptom knowledge. The patient responded that he had no swelling to his feet or belly, but did once upon a time. The total of the interaction confirmed to the researcher that the patient had core knowledge, reinforced by the nurse. A physician asked another patient a similar question about fluid buildup. In this exchange, the patient was asked yes or no questions. Yes or no questions fail to provide sufficient evidence of symptom knowledge. In both cases, the instruction occurred while the HCP was examining the patient's neck veins, hands, abdomen, and feet with no explanation of the rationale for those actions. This would have been a time for HF symptom instruction.

Category 8: Risk modification. AHA Guidelines note that HF risk modification factors linked to smoking, diet, and blood pressure are largely preventable (Skerrett, 2012). Smoking is the leading cause of preventable disease and death. A fruit and vegetable-rich diet coupled with low fat consumption can lower blood pressure (Shi \& Singh, 2011). Modifying health habits, while challenging, can slow disease progression and reduce risk for heart attack, stroke, and HF (MODHHS, 2007). Unlike the universally applicable topics previously analyzed, risk reduction instruction is tailored only to those possessing the risks, which varied among the 10 patients.

Risk modification instruction was marginal, provided to two patients totaling five minutes of observed instruction with a range of 2 minutes to 3 minutes $(M=2.5$; $S D=0.71)$. In the first case, a registered dietician provided three minutes of instruction on smoking cessation to one of the four patients known to be tobacco users; a nurse provided another patient with two minutes of healthy diet instruction on the day of dismissal. The United States Department of Health and Human Services ([USDHHS], 2013) endorses a plan of care that includes content on smoking cessation and a healthy diet to improve quality of life and reduce the risk of hospital admission.Missing instruction included maintaining a specific body weight and maintaining blood pressure. Fluid retention due to acute HF was the main weight challenge of patients during their acute stay. No patient in this study needed weight management instruction beyond fluid restriction. Low blood pressure (82/59) however was a factor for one patient at discharge. While physical activity may lower blood pressure (Beilby, 2004), this patient had exercise intolerance due to HF and low blood pressure due to a discontinued cardiac medication (Milrinone, a phosphodiesterase 3 inhibitor). The nurse told this patient that the extended care facility would receive a copy of the discharge paperwork, suggesting that education would be delegatedto the extended care nurse, and mitigating that the patient also required instruction tied to low blood pressure, syncope, or potential patient safety concerns. One exemplar reflects a disconnect between patient and provider. A registered dietician had a 3 minute interaction with a patient who stated he "lived the trucker's lifestyle including smoking, coffee with five sugars, and no exercise." The dietician stated, "You definitely need to stop smoking." The dietician stated to the patient that the positive effects of healthy eating were offset by smoking. This may not have been an evidence-based statement, but the opportunity to address diet (given the coffee with sugar statement) was observed as a cue for specific dietary discussion. There was no evidence that the dietician provided a referral to a smoking cessation specialist as a form of follow through.

Category 9: End of life. The trajectory for HF patients is one of prolonged illness with HF often listed as the cause of death. For this reason AHA Guidelines include preparatory end of life instruction as part of its instructional standard. This teaching would seem especially important when persistent severe symptoms of refractory end stage HF occur and the treatment options have run out. Yet when presented too early in the trajectory of the disease, end of life instruction may take away hope or create undue stress and anxiety for both patients and HCPs, making the subject difficult to address. AHA recommends discussions on end of life whenever appropriate. Instruction should always occur when the patient is still capable of participating in the 
decision making.Only one patient of the 10 received instruction related to end of life care provided by a physician for one minute. End of life was rarely discussed; one patient requested end of life information and another patient had palliative services at discharge, a service that clinically manages and monitors end of life needs, e.g., pain management (Goodlin, 2009), but no end of life teaching.Some stage 4 heart failure patients could have benefitted from end of life instruction. One patient, with no treatment options left, was offered no comfort care alternatives in what was observed. A social worker from the palliative care team assessed the patient's interests, noting where he lived, if he had social security disability, and whether a durable power of attorney was assigned, carefully listening to each response. In spite of the data gathered, instruction from these queries did not materialize. The line of questioning relates to instructional opportunities, but no provider-patient interaction resulted. An end of life discussion was initiated by a patient who asked of her physician, "How long do I live with heart failure?" This cue was interpreted as patient readiness to discuss end of life issues, yet the subject was not pursued by the provider. Palliative care, with its focus on pain management and how to sustain quality of life, conceivably is a safer place to begin end of life instruction (Goodlin, 2009).

AHA instructional time spent by HCP group. Various providers were engaged in providing HF education. Figure 4.2 reflects the time spent in instruction on the various AHA topics by discipline. Nurses provided the highest percentage of AHA teaching (27\%) compared with the other disciplines.

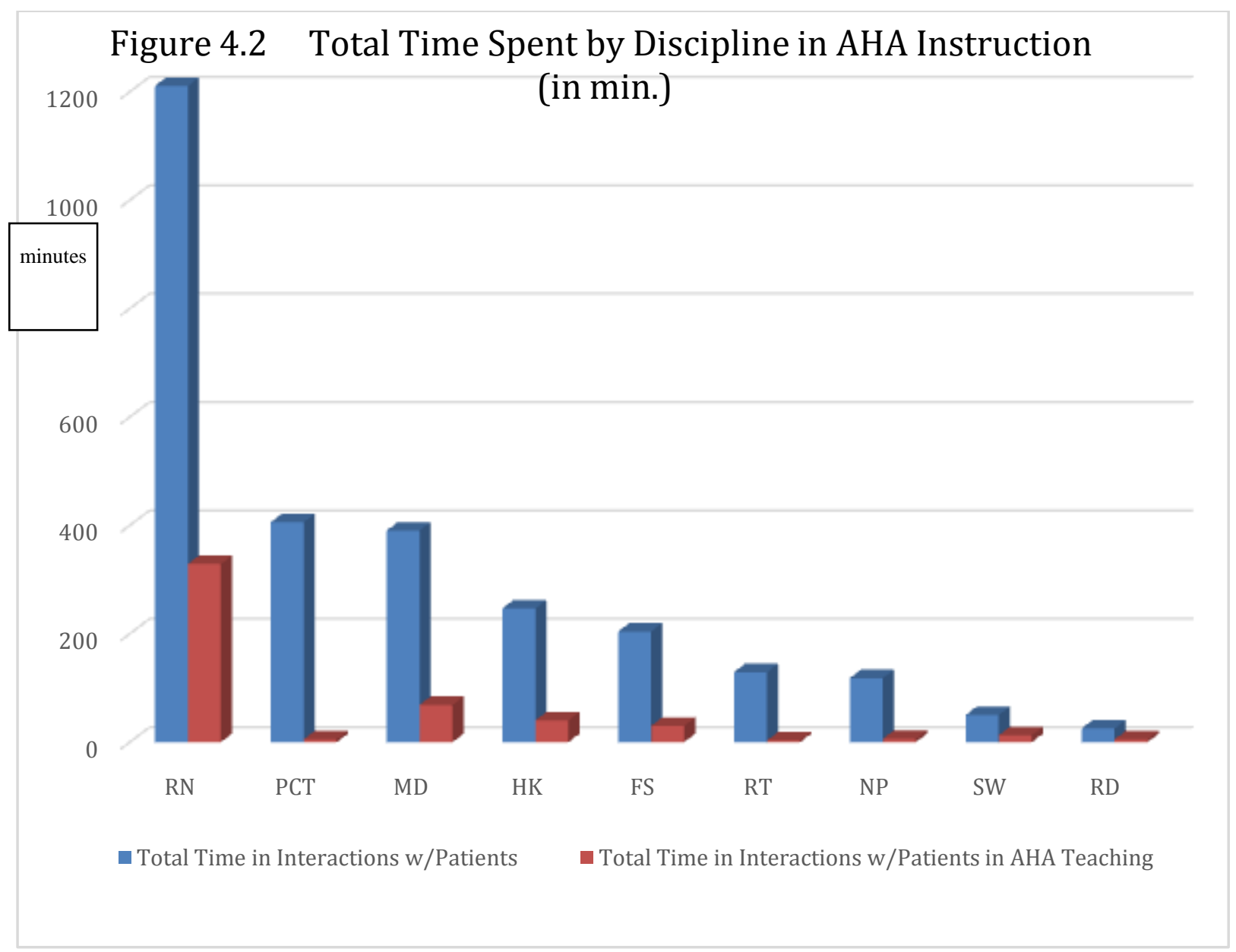

Table 4.4Total Time (min.) of AHA HF Teaching by HF Patient

Total Time for HF Education TotalTime for HF

Recommended per AHA Education $\operatorname{LOS}^{1}\left(\mathrm{D} \mathrm{ICU}^{2}(\right.$

$\begin{array}{llll}\text { Patient Guidelines (60 minutes) (min.) } & \text { ays) } & \text { Days) }\end{array}$

\begin{tabular}{lllll}
\hline A & 60 & $69.5^{3}$ & 10 & 1 \\
B & 60 & $108.6^{3}$ & 6 & \\
C & 60 & 25 & 3 & \\
D & 60 & 19 & 11 & 2 \\
E & 60 & 59.5 & 3 &
\end{tabular}




\begin{tabular}{llll}
$\mathrm{F}$ & 60 & $85^{3}$ & 7 \\
$\mathrm{G}$ & 60 & 42.5 & 4 \\
$\mathrm{H}$ & 60 & 42 & 7 \\
$\mathrm{I}$ & 60 & 35 & 7 \\
$\mathrm{~J}$ & 60 & 12.5 & 1 \\
\hline
\end{tabular}

Note. ${ }^{1}$ Length of stay. ${ }^{2}$ Intensive care unit. ${ }^{3}$ Met standard.

AHA recommends that HF patients receive at least 60 minutes of instruction prior to discharge. Table 4.4 documents whether this standard was met based on the total observed instruction, noting that the researcher was not present around the clock. Three of the 10 patient participants or $30 \%$ of the sample received greater than 60 minutes of instruction.

Sequence of AHA teaching. As part of data analysis, patterns of instruction were examined to identify whether a structure emerged that could guide HCPs as to when to present instructional topics. For instance, many nurses comment that discharge planning is left until patient discharge but currently no data supports this. Based on the data obtained in this study, a "chain" of instruction was developed for each patient using a color coded schema to analyze instructional topics and patterns. Table 4.5 represents an instruction chain with number of minutes of instruction per color. The first color is always the first observed instructionand the last color on the chain is the last observed instruction. The chains are read from left to right and rows are continuous from one row to the next for each patient. For example, Patient $\mathrm{A}$ has three rows of instructions with the first row and first instruction/colorpurple and the last row instruction/color orange.

Table 4.5Observed Chains of HF Instruction (in min.)

\begin{tabular}{|l|llllllll|}
\hline Patient A & 2 & 1 & 6 & 1 & 2 & 1 & 15 & 10 \\
& 1 & 1.5 & 5 & 8 & 1 & 2 & 1 & 1 \\
& 1 & 5 & 5 & & & & & \\
\hline Patient B & 0.5 & 0.5 & 0.5 & 1 & 1 & 1 & 10 & 1 \\
& 10 & 1.5 & 1.5 & 2.5 & 2.5 & 40 & 5 & 10 \\
& 1.7 & 1.7 & 1.7 & 3.75 & 3.75 & 3.75 & 3.75 & \\
\hline Patient C & 5 & 10 & 10 & & & & & \\
\hline Patient D & 14 & 3 & 2 & & & & & \\
\hline Patient E & 2.5 & 2.5 & 1 & 5 & 5 & 12 & 5 & 1 \\
& 3 & 8 & 2.5 & 5 & 2 & 2 & 2 & 1 \\
\hline Patient F & 15 & 2.5 & 2.5 & 10 & 11 & 2 & 10 & 7 \\
& 25 & & & & & & & \\
\hline Patient G & 4 & 4 & 5 & 5 & 1 & 1 & 1 & 3 \\
& 4 & 2.5 & 2 & 2 & 2 & 2 & 2 & 2 \\
\hline Patient H & 3 & 3 & 1 & 2 & 6 & 3 & 4 & 4 \\
& 2 & 2 & 1 & 1 & 1 & 1 & 1 & 6 \\
\hline Patient I & 3 & 3 & 19 & 2 & 2 & 2 & 2 & 2 \\
\hline Patient J & 1 & 0.5 & 2.75 & 2.75 & 2.75 & 2.75 & & \\
\hline
\end{tabular}

Color Code

of Topics:

1
2
3
4
5

Recognition of Symptoms

Activity and Exercise

Medication, Type, Use

Daily Weights

Modify Risks Smoking Cessation

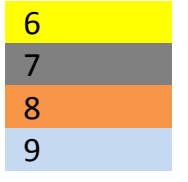

Diet Recommendations

End of Life

Follow up Appointment

Discharge Instructions

QFrom this data, several observations were derived. Table 4.6 presents the number of topics covered categorized as low, medium, or high. In the low range, instructions on medication, diet, and risk modification were provided to Patient $\mathrm{C}$ and Patient $\mathrm{D}$ who received less than 30 minutes of instruction. In the medium range, four patients received instructions on medications, symptom recognition, diet, and follow-up and half of these 
patients received nearly 60 minutes of instruction. The number of topics covered do not equate with time spent. For instance, a variation in time in the medium category from 12.5 minutes to 108.6 minutes was documented.

\begin{tabular}{|c|c|c|c|}
\hline Patient & $\begin{array}{l}\text { Low Range } \\
\text { (1-3 Topics) }\end{array}$ & $\begin{array}{l}\text { Medium Range } \\
\text { (4-6 Topics) }\end{array}$ & $\begin{array}{l}\text { High Range } \\
\text { (7-9 Topics) }\end{array}$ \\
\hline$\overline{A A}$ & - & "69.5 & - \\
\hline B & - & 108.6 & - \\
\hline $\mathrm{C}$ & 25 & - & - \\
\hline $\mathrm{D}$ & 19 & - & - \\
\hline $\mathrm{E}$ & - & 59.5 & - \\
\hline $\mathrm{F}$ & - & - & $* 85$ \\
\hline $\mathrm{G}$ & - & 42.5 & - \\
\hline $\mathrm{H}$ & - & 42 & - \\
\hline I & - & 35 & - \\
\hline $\mathbf{J}$ & & 12.5 & \\
\hline
\end{tabular}

Note. *Discharge Instructions include: symptom recognition, exercise, medications, daily weight, diet, and follow up.

Key observations from the pattern analysis show that medications and symptom recognition are the topics for which instructions are started earliest in the patient's hospital stay and are frequently provided together. Follow up instruction is discussed throughout the patient's hospital stay, thus giving some initial indication that it does not occur primarily at the time of dismissal. There does not appear to be a defined pattern for dietary instruction as it is provided.

Table 4.7MissingAHA Instructionper Patientby Number of Topics

\begin{tabular}{llll} 
Patient & $\begin{array}{l}\text { Low Range } \\
\text { (1-3 Topics) }\end{array}$ & $\begin{array}{l}\text { Medium Range } \\
\text { (4-6 Topics) }\end{array}$ & $\begin{array}{l}\text { High Range } \\
\text { (7-9 Topics) }\end{array}$ \\
\hline \hline A & - & 69.5 & - \\
B & 108.6 & - & - \\
C & - & - & 25 \\
D & - & 19 & - \\
E & 59.5 & - & - \\
F & $* 85$ & - & - \\
G & 42.5 & - & - \\
H & 42 & - & - \\
I & - & 35 & - \\
J & - & 12.5 & - \\
\hline
\end{tabular}

*Discharge Instructions include: symptom recognition, exercise, medications, daily weight, diet, and follow-up

Supplements that support AHA HF education. A benefit of naturalistic inquiry is that it accounts for other variables in the environment that are contributing factors, in the case ofthis study, patient education materials. The most frequently observed teaching method was verbal instruction, but print materials in the form of brochures and handouts were also used. These were available in the lounge for family access or presented to the patient by the HCP as a teaching supplement. Written materials included Pump Up Your Heart: A Guide to Understanding Heart Failure, Heart Failure, Cigarette Smoking and its Health Risks, Stay Healthy Outpatient Program (SHOP), other dietary brochures, a fluid measurement sheet, and discharge paperwork.

The most comprehensive teaching guide was a hospital-developed pamphlet, Pump Up Your Heart. In this colorful 35-page, 18 font brochure, data revealed the content matched with eightcategories of the AHA Guidelines, yet it was not used by HCPs to guide their teaching. One nurse was observed teaching a patient about the importance of daily weights and showing a patient the weight record within the brochure to record a daily weight. Had this teaching tool been used with patients, a significant improvement in meeting AHA standards would have been met.Enhanced HF teaching on AHA content helps patientsaquire knowledge, strategies, and skills necessary to stay motivated and adhere to treatment plans and self care.

The brochure presents HF medications, the reason for being prescribed, and information on obtaining medication refills. Specific drugs are addressed including angiotensin-converting enzyme (ACE) inhibitors, 
angiotensin II receptor blockers (ARBs), Beta Blockers, Diuretics, and Digoxin. Next, the importance of a low sodium diet is explained with foods to avoid, an introduction to counting sodium by milligrams, and practical ideas that would allow the patient to make "better choices" at each meal. Risk factors are addressed, such as who to contact for support in stopping smoking. Healthy activities/exercise are explained including how to increase the length of exercise each week by five minutes until reaching the exercise goal of 20 minutes. Absent is advice on exercises for warm up and cool down. Using the reading level assessment tool, it was determined that the brochure was written at or above the sixth grade reading level, which shows consideration of literacy levels ("Readability," n.d.). The next artifact analyzed wasStay Healthy Outpatient Program (SHOP), a onepage guide providing instruction on the post-hospital services that are available to patients. The information on this one page guide includes medication programs, transportation, and problem solving services through weekly phone calls and home visits that can be accessed for up to two months. High risk patients for readmission are identified and an assessment conducted by a nurse or social worker. The guide met one AHA Guideline for the follow up category. Only one patient received a SHOP guide which resulted from an assessment conducted by a social worker.

Several food and nutrition brochures were discovered in the study setting which conform to AHA Guidelines tied to dietary instruction. At no time during the observational time were these documents used. Two brochures, A Guide to Sodium Content and Low Sodium Shopping Guide, published by the hospital's Heart and Vascular Center, were found in the lounge on one acute cardiology unit. Each brochure included shopping tips that went beyond the AHA Guidelines, such as why one might buy the store brand, searching for costeffective items on the bottom shelf, and buying in bulk. The Low Sodium Shopping Guide identified several local grocery stores by name and listed food items with the sodium content in milligrams that can be found within that store. The Guide to Sodium Content brochure listed food groups (fruits, vegetables, meats, dairy, grains and cereal, snacks, drinks, seasonings and condiments, fats and oils) with multiple examples of foods along with low sodium shopping tips and budget tips.

A third heart healthy eating tool was given to one patient, generated from a computer-printout. Written at or above the seventh grade level, content was not well defined (such as explaining what constituted a triglyceride before launching into making food choices about triglycerides; "Readability," n.d.). Another observation was that the material contained in this printout was always from a negative view, cautionary foods, but with no positive examples of desirable options. This handout met two AHA Guidelines related to eating a well-balanced diet and following a low sodium diet. No dietary handouts were discovered that could be tailored to a patient's specific needs.

Another tool that was not used during the investigator's observations was the Heart Failure Traffic Lights. The model for this tool was the common traffic light. The content of this brochure covered five AHA categories: symptom recognition, activity/exercise, daily weight, low salt diet, and follow up appointments. The handout was found in the family waiting area yet no HCP reviewed the handout with a study patient nor was the handout found within a patient's room. In the topic of symptom management, the green light listed symptoms being "all clear" for the absence of shortness of breath, swelling, weight gain, and no decrease in activity level. The yellow light listed cautionary symptoms such as shortness of breath, weight gain, cough, ankle/foot swelling, wheezing, chest pain, need for increase in pillows to sleep, decreased energy, and lightheadedness. The red light listed symptoms for medical alert such as unrelieved shortness of breath, new onset of chest pain, dizziness that would prohibit the patient from standing up.

Finally, a Cigarette Smoking and its Health Risks handout produced by the hospital was present in the patient waiting area. No HCP was observed using this material to teach four patients who had used tobacco for most of their lives, nor was this handout found in the patients' rooms. The material covered smoking and heart and lung disease, gastrointestinal disease, and other smoking-linked disorders. The content on pathophysiology was at a $10^{\text {th }}$ grade reading level and met the AHA guideline related to risk modification on smoking cessation. Additionally, the handout reviewed women and smoking, passive smoke, chewing tobacco, and quitting tips. Content absent from the handout included information on electronic cigarettes and vaping.

Non-instructional print materials were also noted. The fluid measurement handout is a one-page sheet containing a list of 14 beverages (coffee, tea, milk, soda, etc.) and iced treats that are available to patients who are on fluid restrictions in the hospital. These items were randomly listed, in no specific order, i.e. from largest to smallest quantity or alpha sorted by name of item. The measurement sheet could not be individualized to a patient's needs nor were there examples of the different fluids or iced treats that totaled the specific fluid restriction amount, i.e. $1500 \mathrm{cc}$ equals one cup of tea, one glass of milk, and one pitcher of water for sipping throughout the day.

As part of the documentation provided at patient dismissal, forms are produced that containseveral pages of instruction. This form covers six AHA Guideline categories as noted earlier along with other topics incongruent with the patient experience (i.e. immunizations, pneumonia screening, cardiac rehabilitation, and mental health care). 
Research Aim 2 (RA2): Non -AHA HF education provided during the acute care stay. The research questions related to aim 2 were: (a) What content fell outside of the AHA Guidelines? (2) Which HCPs provided HF instruction outside the guidelines? (c) How much time was spent on this HF teaching? The major finding that fell outside of the AHA Guidelines was the significant amount of time spent providing instruction in the current plan of care (CPOC). The compromised health status of each patient, the active treatment shifts tied to re-stabilizing the HF condition, and complicating factors from secondary diagnoses all required instruction aimed at the patient. Of the 911.1 minutes of instructional effort observed in this study, 412.5 minutes of educational effort were related to the current plan of care. Each day, instruction tied to the CPOC occurred for all patients $(n=10)$, with time spent ranging from 9 to 142.5 minutes during a length of stay. Physicians were frequently engaged with this instruction (223.5 minutes) provided when examining the patient.

\begin{tabular}{|c|c|c|c|}
\hline Patient & $\begin{array}{l}\text { Total Minutes } \\
\text { in } \mathrm{CPOC}^{1}\end{array}$ & $\begin{array}{l}\mathrm{LOS}^{2} \\
\text { (Days) }\end{array}$ & $\begin{array}{l}\mathrm{ICU}^{3} \\
\text { (Days) }\end{array}$ \\
\hline A & 142.5 & 10 & 1 \\
\hline B & 11 & 6 & \\
\hline $\mathrm{C}$ & 28 & 3 & \\
\hline D & 73 & 11 & 2 \\
\hline $\mathrm{E}$ & 21 & 3 & \\
\hline $\mathrm{F}$ & 6 & 7 & \\
\hline G & 16 & 4 & \\
\hline $\mathrm{H}$ & 73 & 7 & \\
\hline I & 33 & 7 & 2 \\
\hline $\mathbf{J}$ & 9 & 1 & \\
\hline Total & 412.5 & 59 & 5 \\
\hline
\end{tabular}

Note. ${ }^{1}$ Current Plan of Care. ${ }^{2}$ Length of Stay. ${ }^{3}$ Intensive Care Unit.

The instruction provided varied by patient and the immediate health condition. For instance, one patient admitted for a second opinion was assessed by a team of physicians. The attending physician asked questions related to the patient's other recent HF admissions, his ability to breathe, and valve (mitral or atrial) involvement. Five different physicians listened to the patient's heart sounds as the attending physician instructed, "In a day or so, we'll place a plastic tube in your heart then decide the best medicines for you." Another physician obtained consent for the anticipated procedure and explained that through this procedure, "We can see the severity of [your] heart failure, measure the pressures in the heart chambers, [and perform a] Dobutamine evaluation." The Dobutaminediscussion became a topic of further CPOC instruction. The researcher noted variability in instruction, from the simple, "tube in your heart" to the very complex "Dobutamine in your chambers" discussion with post-discharge implications. There were unanswered questions which led to patient and family anxiety. Further noted was that throughout the hospital stay the patient could not pronounce the drug's name.

Another patient received CPOC instructions on kidney function, the physician instructed the patient that her "kidneys were going up and down. This is a sign of the heart getting weaker and this is what you can expect." The researcher noted instruction of the patient's "kidney's going up and down" could have multiple meanings and the patient did not ask clarifying questions. The HCP did not validate the patient's understanding of what she heard.

The findings on non-AHA and AHA HF instruction showed that $10 \%$ more time was spent on AHA HF teaching than on non-AHA teaching $(\mathrm{AHA}=498.6$ minutes; non- $\mathrm{AHA}=412.5$ minutes). $\mathrm{CPOC}$ was fundamental to the HF patient's acute care stay and was identified as HF education topic that went beyond AHA recommended guidelines. Nurses spent the most time providing AHA instruction (328.6 minutes) thanother $\mathrm{HCP}$ groups and physicians spent the most time providing HF instruction on the CPOC (223.5 minutes) than other HCP groups. The AHA HF guidelines are intended to assist the HCP with a range of patient education topics, however, the overall data illustrates that time spent on non-AHA HF teaching competes closely with AHA instruction. However, not all AHA Guidelines are utilized in the acute care setting. Results from the expert HCPs surveys are presented in response to Research Aim 3 (RA3) in the next section.

Research Aim 3 (RA3): Expert HCPs identify critical HF education for patients experiencing an acute episode of HF.The research questions for aim 3 were: (a) Where was the congruence between HF Guidelines and expert recommendations/instruction? (b) Where was there incongruence between the HF Guidelines and expert recommendations/instruction? More in-depth information from expert providers, HCPs with expertise in HF management, is presented in this section. Twenty-nine surveys were distributed and 12 expert HCPs 
responded (41\% response rate). The expert HCPs responded to three questions below. To ensure that their responses did not influence the usual HCP and HF patient interaction, surveys were distributed after data collection for RA1 and RA2 was complete. The findings related to RA3 are detailed below.

HF teaching most appropriate/important from the expert HCPs experience. The most frequent responses to most important HF teaching included patient education on medication $(\mathrm{n}=7,58 \%)$ and $\operatorname{diet}(\mathrm{n}=8,67 \%)$ compliance. These findings are consistent with two of the nine AHA categories found in RA1.Physicians noted their patients takingmedication as prescribed and understanding how these medications would keep "their heart functioning as well as possible for as long as possible" were most important. Physicians also expressed concern about patients' knowledge on the relationship between diet and fluid retention. These expert HCPs explained the importance of teaching to each patient's need. Expert HCPs named with less frequency the importance of instruction pertaining to daily weight $(\mathrm{n}=4,33 \%)$, symptom recognition related to shortness of breath and follow-up appointments (both $\mathrm{n}=2,17 \%)$ and fluid retention $(\mathrm{n}=1,8 \%)$. AHA topicsmissing from the expert survey responses included exercise, risk modification, and end of life teaching related to refractory end stage HF. The expert HCPs may have omitted theseareas due to their patients' HF-related exercise intolerance, absence of risks factors, avoidance of the topic (i.e. end of life), reliance on team members to address HF teaching, and addressing the urgent needs of the patient, i.e. CPOC.

Benefits of HF teaching to HF patients. Expert HCPs identified the benefits of HF teaching in the acute care setting. One nurse practitioner responded, "Keeping them [HF patients] out of the hospital and understanding medications and benefits of medications." A physician noted, "Recognition of symptoms of heart failure decompensation or progression ... understanding of the natural history of their disease and what challenges may lie ahead."

Several expert HCPs identified that knowledge of the disease, the disease process, or pathophysiology would benefit the HF patient. One physician stated, "Many times [patients] never have been told what their disease even is or what it means in terms of their future quality of life or mortality, so it's not something they take as seriously as they should." Another physician wrote, "The more a patient understands their disease, the better they take care of themselves." Expert HCPs made no mention of health literacy or cultural beliefs or practices related to teaching patients about heart failure, its trajectory, treatment, and management, topics supported by Campinha-Bacote (2007) and Parker and Ratzan (2010).

New clinical issues or trends expert HCPs see changing the nature of or the content of clinical education. Expert HCPs survey responses related to clinical issues or trends changing the content of clinical education specifically cited HF education, pathophysiology, and the process of the disease. One nurse practitioner wrote, "CHF education would be critical in preventing readmissions to hospital for exacerbation. If patients understand pathology, med regime, and disease process of CHF then ideally they could manage their heart failure better; reduce readmission rates." Also, half of the expert HCPs mentioned trends in technology, on-line apps, mobile technology, and advanced treatments. One physician noted, "More and more advanced treatments are coming out which even a lot of physicians don't completely understand. Once a patient fails medical management, they need to understand what other options exist home infusion, LVAD, transplant." While these statements were visionary, they stood out as incongruent with the teaching methods used at the bedside mostly verbal communication and sparse use of handouts and brochures. While the use of technology was not observed, it may be that traditional print materials are no longer as relevant in today's technology driven world.

\section{Expert Suggestions for HF Teaching}

Two significant themes outside of AHA Guidelines were identified, both were non-topical in nature: the desire for a therapeutic alliance between HCPs and the patient and the use of new technologies for monitoring and treatment. Because HF is a progressive disease, effective HCP and HF patient educational encounters are fundamental. One expert explains, "A therapeutic alliance [between the HCP and HF patient] is essential." Another expert states, "This [knowledge about self-care management] can keep the patient out of the hospital and help them live longer." A therapeutic alliance must be patient centered, cost effective (Pugh et al., 1999), and utilize AHA "teach to goal" principles that incorporate multiple HCP encounters that evaluate patient learning and focus on simplified instruction.

There are important trends on the horizon, such as multidisciplinary care outpatient services (McDonald et al., 2002), medical homes (USDHHS, 2014), and tele-health (Radhakrishnan\&Jacelon, 2012). These services closely follow HF patients in the outpatient setting replacing episodic disease-focused care with a promising new therapeutic alliance that facilitates self-management strategies in the outpatient setting. One expert states, "Advanced therapies are a double edge sword; [procedures or surgeries] benefit the patient, but CHF is a terminal disease." Scientific and technological advances can create public demand for services which in turn impact productivity, eliminate certain illnesses, and increase longevity. The Expert HCPs (physicians, nurse practitioners, and nurse educators) must critically review the scientific, technological advances, and emerging trends, and be at the center for policy changes and recommendations for transformations in health care 
delivery. The key focus expressed by HCPs is relationship-centered instruction and the potential use of technology to deliver and reinforce education in real-time based on the patient's condition.

\section{Themes}

Four key themesidentified in Chapter 2 from the literature were $H F$ education, patient experience, coordination of care, and HF Guidelines. They were well represented in the contiguous account of providerpatient interactions as described in the preceding sections.Yet other themes emerged from provider - patient exchangesincluding "patient disposition" and "patient need for hope"(see Appendix G).

\section{Summary}

In this study, HCP and patient encounters were observed by the researcher with as little imposition as possible in order to allow for the normal flow of care delivery. The data collected were based on direct observation, a collection of teaching artifacts, and documented field notes. Patients received HF teaching most frequently from physicians and nurses on topics that addressed medications, symptom recognition, diet, and follow-up appointments. Supplemental brochures and handouts were written at varying literacy levels and covered many of the guidelines suggested by the AHA, with exceptions previously documented. Instructional interactions varied widely by patient and many inconsistencies were noted related to assessing the patient's literacy level, readiness to learn, and ability to act on instructions. Opportunities for instruction were missed, most notably when tasks such as performing a physical examination or during medication administration occurred. A major finding was that the current plan of care was a major trigger for instruction and a topic not addressed in AHA Guidelines. An expert panel confirmed topics noted in these guidelines as relevant, with the most important topics identified as medication and diet instruction. Additional emerging themes were also identified.

\section{Discussion, Implications, \& Recommendations}

\section{DISCUSSION}

In this chapter, a summary of the research findings, how the research relates to other literature, the implications of the findings, and recommendations for further research are presented. This study achieved its purpose: to ascertain whether HF patients received HF instructioncongruent with or beyond the AHA Guidelines,how instructional information was delivered and how patient's responded to this instruction,and identification of HF topics considered as critical for HF instruction.

Naturalistic inquiry brings with it unique themesparticular to that real life setting. Two such themes that emerged were patient disposition and patient need for hope (CPOP is discussed under the Research Findings section). The strongest patient responses were met with actions. For example, patients who became upset due to delays in procedures or discharge, receiving bad news related to their health, or conflicts with a roommate were consistently attended to by sensitive, compassionate HCPs. These themes are relative to this acute care setting but likely to be morein the vanguard of conflict resolution or hope intervention research.

Sixty-nine HCPs provided HF instruction to a sample of 10 Stage 3 and $4 \mathrm{HF}$ patients. Field notes were recorded to capture the details of the interactions between HCPs and patients and the context-rich intricacies of instruction. Capturing these details is thestrength of naturalistic inquiry as a framework for fieldbased research. Artifacts, such as printed instructional guides, were collected and analyzed to identify nonverbal instruction.

\section{Research Findings}

The AHA Guidelines are the national standard for providing HF education to patients within the hospital setting. Research on use of these standards in the practice setting showed that they are comprehensive and useful as verified by an expert panel of HCPs. In this study, the AHA Guidelines were noted to overlap in some categories; each of the nine topical areas is not mutually exclusive. Further, many of the standards applied to all patients, but those that addressed lifestyle and end-of-life issuesare subjects that may or may not apply to individual patients, or are time-sensitive in the patient's disease trajectory. HF education observed in the practice setting readily fit into AHA subject categories, with the following findings.

First, as comprehensive as the AHA Guidelines are, not all topics were consistently covered. The premise of this study was that HF education is a critical intervention linked to disease management, self-care capacity, and prolonged quality of life. Ultimately, instruction that leads to patient engagement in managing $\mathrm{HF}$ can delay or prevent hospitalization, a signal that the disease is progressing. 
The overall time spent in HF education was less than one hourfor $70 \%(n=7)$ of the patients observed during this study. Although data were collected during times of high HCP - patient interaction and for each contiguous day throughout the patients stay, it was not done around the clock, a study limitation. Seventy percent of HF patients observed received a range of approximately 12 to just under 60 minutes of instruction compared to the AHA standard of sixty minutes per patient per stay. Thirty percent $(n=3)$ of HF patients exceeded this standard, receiving approximately 70 to 108 minutes of instruction.

While the AHA Guidelines are considered comprehensive, this study aimed to discover whether there were gaps in the guidelines. A second major finding was that AHA Guidelines did not include the considerable instructional time spent teaching about the current plan of care (CPOC) that the patient is undergoing. Acute $\mathrm{HF}$ patients experience multiple kinds of treatments, ranging from diagnostic to therapeutic, such as medication adjustments. The time spent on CPOC instruction nearly equaledthe time spent on AHA topics. CPOC instruction is currently not part of the AHA Guidelines.

An unexpected third finding (also considered a gap) was the rare use by HCPs and patients of printededucational resources such asbrochures and handouts specifically designed for HFpatients. Content analysis revealed that the topics in these materials were not inclusive of the AHA standards. However, had they been used, three AHA topics tied to exercise, physiologic changes to expect during exercise, and refilling prescriptions would have increased topical coverage from $50 \%(\mathrm{n}=14)$ to $61 \%(\mathrm{n}=17)$. The AHA Guidelines would have been a useful organizing framework for developing more complete print and other support educational materials. Still, given the usefulness of the current materials as a way to standardize education, it would be reasonable for HCPs to offer, use, or encourage their use.

Another finding surfaced when examining the sequence of education provided. Four areas stood out as instructional priorities, reinforced by the expert panel's survey comments, and congruent with AHA Guidelines: medications, symptom recognition, diet, and follow-up appointments. Coverage of these topics was found to be spread out over the hospital stay.

The last major finding relates to $\mathrm{HCP}$ - patient interactions. The disciplines who delivered the most instruction (AHA or non-AHA) were physicians and nurses. Significant was that patient knowledge and literacy was not assessed or considered when delivering education. There were few examples of intentional/focused instruction. Rather, the typical instruction provided was piecemeal in both topics covered, with no one overseeing how the instruction and the patient's response to teaching was evolving. The urgency of carrying out medical procedures and stabilizing the patient's condition led to instruction about the current plan of care with less concern for how the patient would have the ability to self-manage after dismissal. Several topics seemed off limits for instruction because of their clinical complexity. For instance, the subjects of dry weight and its calculation, and the patient's assessment of heart rate as normal versus abnormal were not discussed. It may be more useful to broach the subject of palliative care in the practice setting and consider changing the AHA Guidelines accordingly.

\section{Relationship of the Study to Other Literature}

A review of the published research literature provided contrast and comparative information. The corpus of data on HCP and HF patient instructional interactions areaugmented with two bodies of literature: patient centered care and effective communication.

\section{Patient Centered Care}

Patient centered care includes incorporating the perspective, needs, and wants of the patient in the clinical experience, where patient input and participation in their care and partnering with the HCP occurs (Ishikawa, Hashimoto, \&Kiuchi, 2013). A patient centered approach grants the patient an active voice in receiving care, rather than the repository of $\mathrm{HCP}$ advice. HF instruction should have patient-centeredness at its core.

Limited patient-centeredness was observed throughout this study. For instance, one patient had little power or control during a physician interaction due to time pressure and perceived urgency for the physician to move along, offering the patient no opportunity for feedback. This anxious patient first interacted with the physician who stated, "So you're not taking your medicines?" and who then went on to discusstest results and treatment options. There was no discussion of whether the patient could afford medicines or needed help in setting up his daily medications. The physician's use of language showed dominance over the patient. As noted by Anderson (2012), words can empower or degrade but are never neutral.

\section{Effective Communication}

Joint Commission and other government and health organizations endorse best practice standards that include culturally and linguistically effective communication strategies between providers and patients. Providers are challenged to strengthen their knowledge, attitudes, and behaviors within healthcare organizations 
toward populations that are increasingly diverse (HHS/OMH, 1991). Communication is central to patientcentered care, but also provides the opportunity for continuous feedback, where providers and patients interact, reflect, and evaluate their priorities, understanding, and clinical approaches. This iterative style of communication is a key facet for patient participation and full engagement. Elwyn and coworkers (2003) devised a framework to advance HCP and patient communication that included presenting the problem, formulating the treatment options and identifying risks, exploring patient concerns and checking patient understanding, and providing opportunity to defer a decision. The HF patients in this study could have benefited from this communication framework. Aversion to the responsibility of effective provider - patient communication may perpetuate the status quo.

Ineffective communication in the context of health literacyimposes barriers to better self-care ability and increases the risk for poor outcomes and hospitalizations (Peterson et al., 2012). As an example in this study, when a patient needed an IV infusion of a powerful cardiac medication post-discharge, it was the patient who assured the HCPs on several occasions that his rehabilitation center could handle his needs because the head nurse used to work in the emergency room. The HCPs knew that for the rehabilitation center to be reimbursed for IV therapy strict criteria must be met, but this information was withheld from the patient. When the patient was informed he could not return to rehabilitation, he lost hope and ended up being discharged to palliative care without IV medicine. This example reflects on communication as a link to trust and psychological preparedness for the future.

\section{Implications and Recommendations}

There are several implications of this study. First, few studies are designed using naturalistic inquiry and this study shows the richness of examining the full context of HF education through deep immersion. While the results of a study of this nature are not generalizable, the findings provide a rich foundation for additional study. Federal agencies such as the Agency for Healthcare Research and Quality (AHRQ) and professional organizations similar to the American Heart Association have issued practice-based guidelines based on the best available evidence. The AHA Guidelines areevidence-based, and the review of the literature conducted for this study reinforced the richness of evidence used by AHA. Guidelines should be used to develop educational approaches that encompass patient-centered care and iterative patient - provider communication. Studies similar to this in areas such as oncology, orthopedics, and other clinically-based specialties would show where gaps in education exist so that guidelines for patient education in other specialties can be developed including designing patient education materials.

Teamwork is touted as essential for clinical outcomes. Patient instruction is assumed to be led by the team. The gaps of education in content, consistency of information, and provider-to-provider interactions put the burden of understanding and synthesizing sometimes disparate information on the patient's back. In HF, this becomes high risk for the patient and for the hospital's reputation tied to public outcome reporting and reimbursement.

Guidelines must be usable and clear. Whether the AHA Guidelines represent minimal standards or optimal standard of practice remains unanswered. The topics within the guidelines required a level of clinical acumen that would be unreasonable for many patients to achieve on their own, such as dry weight calculations, or discerning heart rate as a critical symptom. One sub-topic, patient literacy, was not an area of instruction, but rather an activity to be performed by the HCP. The end-of-life topic should be reframed for broader acceptance and linked to palliative care.

In this study, there was an obvious investment by the hospital in instructional printed guidelines, but in a technology-driven age, the use of applications tied to phones or tablet devices may be a better investment. Technology enhanced devices can reference a dictionary, change the font size, and even accommodate various languages. With the rapid uptake of clinical information, there is less cost associated with changes that may be made to content. Applications can also be designed to be patient-centered and customized.

Another implication of this study is linked to secondary diagnoses. While each patient was instructed on HF, patients had multiple secondary diagnoses, so using a single set of teaching guidelines would miss other confounding instructional needs. The need for evidence-based standards and standardized approaches to instruction must be counter-balanced with individual instruction that can be linked to other diagnoses, such as diabetes, hypertension, arthritis, cancer, and other conditions. The implication is that standards need to be tailored and clinicians need to develop an instructional plan that reflects tailoring to the presence of other conditions.

A final implication is that this study linked clinical and organizational science together. The focus of this study was on HF patients, but the reality is that the study revealed much about job roles and responsibilities as designed for an organizational setting, showed work flow, and how instruction did or did not align with the perceived work of clinicians, and demonstrated that many organizational connectors between services, departments, and pre- and post-hospital connections were all linked into the performance outcomes. The SHOP 
program was an example of an important post-hospital service specifically designed for HF patients. The discharge planning instruction sheet was designed to capture and centralize teaching for post-hospital success. The design of these programs and structures were as much about catching the gaps in the design of job roles and organizational processes as it was about meeting patient's needs efficiently and effectively. Acute HF is a chaotic time for the patient and those trying to accommodate needs. For this reason, research that can reveal some longitudinal perspectives on effectiveness in meeting patient needs and outcome offers huge insights into organizational performance.

\section{CONCLUSIONS AND RECOMMENDATIONS FORPRACTICE AND RESEARCH}

The findings from this study should illuminate a better understanding about HCP and HF patient interactions in the acute care setting as it relates to HF teaching. The best, most effective and meaningful HF teaching must include effective communication and patient centered care during HCP and HF patient exchanges. Patient and organizational outcomes are compromised without effective and efficient HF instruction.

Based on this preliminary study,one recommendation isto expand the AHA guidelines to include topics linked to the CPOC. A second recommendation is for organizations to examine the use of high quality clinical guidelines when developing an intentional approach to patient instruction. This would ensure a more comprehensive coverage of important information, determine which discipline owns the instructional responsibility for addressing a topic, and ensure that various providers who are close to the patient assess and determine the appropriate level of instruction to the patient's level of comprehension.

A third recommendation is for instruction to become normalized in every patient - provider interaction. Whether performing a physical examination, or administering medications, all HCPs should teach. The AHA guidelines are a legitimate framework for providing instruction about the disease, treatment options, symptom management, medications, and their impact, exercise and the like.

Next, all patients should have an assessment for literacy that is used by all HCPs. Feedback to HCPs on the appropriateness of teaching to literacy level, of recognizing cultural and of knowing socio-economic realities, and where the patient will be discharged to (home, another health facility, etc.) should be part of peer reviewed feedback. All health professional schools should incorporate patient instruction into curricula and clinical experiences.

The next recommendation is for organizations to conduct studies that link clinical and organizational outcomes. These studies must be immersive and longitudinal in order to capture work flow and design, and should account for multiple variables in a naturalistic setting. Deep knowledge of operations, roles, and functions are revealed when examining the larger context of care delivery. This study deserves replication in other settings, such as a community or rural hospital environment. Other clinical topics with defined guidelines should be studied and compared to the results of this research from an organizational perspective. Research designed todetermine pre- and post-hospital instruction would reveal the consequences and impact of HF patient outcomes linked to hospital-based instruction. These studies should be designed to determine the preferred sequence of presenting information so that instruction can be scaffolded from easier to grasp instruction to instruction that deals with highly complex and nuanced clinical situations that patients are expected to manage. In conclusion, this study shows the context of where and how HF instruction is delivered to high risk, advanced stage HF patients. The AHA Guidelines are comprehensive but insufficiently referenced in this one setting. Expert HCPs agreed with the "core four" of topics that must be addressed, including medications, symptom recognition, diet, and follow up. The findings of a study of this nature are not generalizable, but still important for future research development. Testing HF education in other acute and non-acute settings may complete a more robust picture of the benefits of structured versus just-in-time instruction. HCPs would benefit from including instruction in each and every patient - provider interaction. The results of this study should create an impetus for other comprehensive patient education instructional strategies.

APPENDIX A

Patient - Provider of Care Observation Protocol/Tool Patient Identification No.

\begin{tabular}{|l|l|l|l|l|l|l|}
\hline $\begin{array}{l}\text { Initials of } \\
\text { Provider }\end{array}$ & Discipline* & Content** & $\begin{array}{l}\text { Method } \\
\text { (verbal } \\
\text { diagram)** } \\
*\end{array}$ & Time Spent & $\begin{array}{l}\text { AHA } \\
\text { Guidelines**** }\end{array}$ & $\begin{array}{l}\text { Comment } \\
\text { s }\end{array}$ \\
\hline & & & & & \\
\hline
\end{tabular}

*NP, RN, MD, CM, SW, RD, PT, OT, ST, Pastoral Care

**Meds, Procedural, Dz (disease), Dx (diagnosis), other

***V (verbal), P (printed), D (diagrams), VC (video) 
****RS (recognition of symptoms); A (activity/exercise); MA (medication adherence); DW (daily weights); MR (modify risks for HF); LSD (low sodium diet); EOL (end of life); FU (follow-up appointments); DI (discharge instructions) 


\section{APPENDIX B}

Identification No.

Heart Failure Content Survey

Congestive heart failure (CHF) patients have been studied and we know that education is an important aspect of treatment. As an expert in heart failure (HF), please answer the following questions:

1) When thinking about the broad nature of HF education, it is presumed that not all education can be done in the hospital setting. In thinking about your patients, what HF education is most appropriate/important from your experience?

2) What do you see as the benefit of HF education to patient?

3) What new clinical issues or trends do you see as changing the nature of or content of clinical education?

\section{APPENDIX C}

Participant Demographic Information

This demographic information will be obtained from the medical record and stored in a locked cabinet, available to the primary investigator only.

1. What is the participant's sex?

\section{Male}

Female

2. In what YEAR was the participant born?

With which ethnic/racial group does the participant most identify?

American Indian/Alaska Native

Asian

Black/African American

Native Hawaiian/Pacific Islander

White

Other

4. Nursing Unit (please circle one):

A $\quad$ B $\quad$ C D

\section{APPENDIX D}

Experience of Knowledge Acquisition of Heart Failure Patients

Model of Analysis

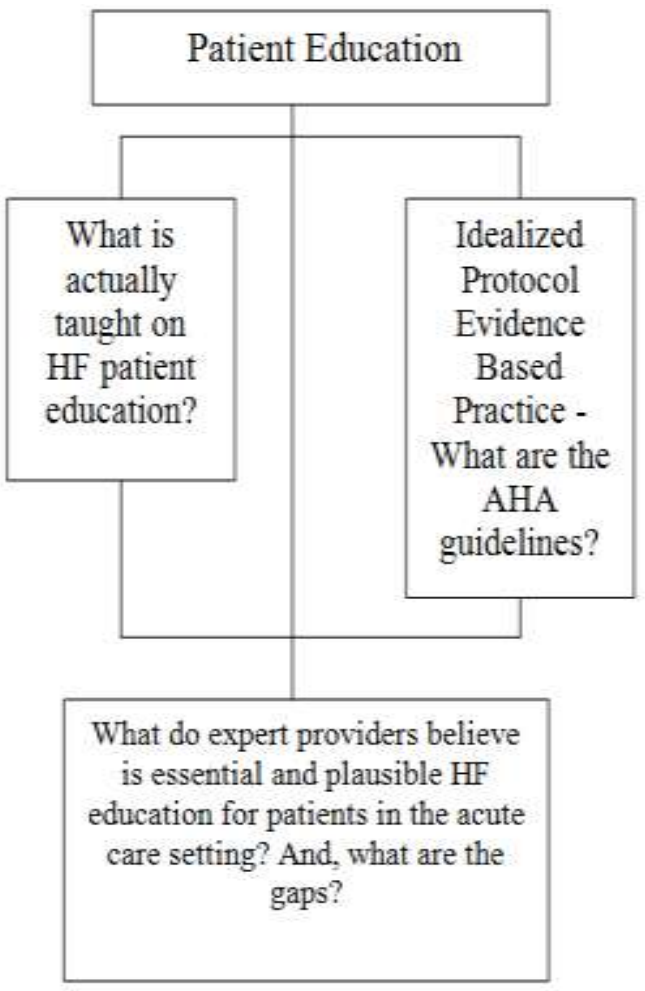




\title{
The Experience of Knowledge
} Acquisition of HF Patients in an Acute Care Setting

\author{
Who: Patients with HF (NYHA III - IV, age 50 -80) \\ When: April - June 2014 \\ (From Admission until Discharge) \\ Where: UNITS A, B, C, \& D at \\ MIDWEST HOSPITAL \\ Hospital Drive

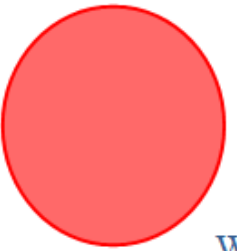 \\ City, State 12345 \\ Why: To examine patient - provider exchanges of \\ information, with the results beneficial to you and the \\ provider team to help understand the types and substance \\ of exchanges that will inform the team of a particular set \\ of strengths and potential opportunities for improvement

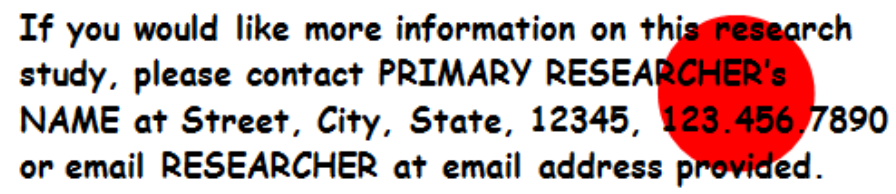

\section{APPENDIX F}

HF Education Themes and Categories from the AHA Guidelines

1. Recognition of escalating symptoms and concrete plan for response to particular symptom:

a. SOB

b. Persistent coughing/wheezing

c. Build-up of excess fluid in the body

d. Tired, fatigue, decrease in exercise and activity

e. Lack of appetite, nausea

f. Increased heart rate

2. Activity/exercise recommendations

a. How to carry out exercise/activity

b. How long to carry out exercise/activity

c. Expected physiological changes with exercises (moderate increased heart rate, breathing effort, diaphoresis)

d. Type and length of time completing warm-up exercises

e. Type and length of time completing cool-down exercises

3. Indications, use, and need for adherence with each medication prescribed at discharge

a. Reiterate medication name, dosing schedule, basic reason for specific medications, expected side effects, and what to do if a dose was missed

b. Literacy assessment: ask patient to read/interpret instructions from a prescription bottle or procedure preparation instruction

c. Ensure the patient has a plan for refilling medications on time

4. Importance of daily weight monitoring

a. Obtain daily weight 
b. Compare today's weight with "dry" weight

c. Normal and exceptional limits of weight gain

d. Actions to take when weight increases above set limits (fluid overload)

5. Modify risks for $\mathrm{HF}$ progression

a. Smoking cessation (counseling/pharmacological therapy)

b. Maintain specific body weight i.e. obesity and insulin resistance, blood pressure, heart function

c. Provide practical information about eating a well balanced diet and promoting normal body mass i.e. high in fruits/vegetables and low in saturated fats and non-whole grain carbohydrates

d. Maintain blood pressure in target range

6. Specific diet recommendations

a. Sodium restriction: how to read food labels checking sodium per serving; sort foods into high sodium and low sodium groups

b. Alcohol limit or need for abstinence

7. Discussion on options for end of life care in patients with severe symptoms/refractory end stage HF

8. Follow-up appointment to improve quality of life, decrease readmission: location, date, and time

9. Discharge instructions, educational materials that address all domains especially diet, medicines, activity, follow-up appointments, daily weight, what to do if symptoms worsen.

\section{APPENDIX G}

HF Education Themes and Categories Beyond AHA HF Education

10. Plan of care

a. Relaying current plan of care to patient

1. What does patient know about current plan of care/information given to patient

2. NPO status

3. Medical/social history requested

4. Code status/Durable Power of Attorney (DPOA)

b. Asking patient for information about plan of care

c. Refused plan of care

1. Activity

2. Test

11. Patient disposition

a. Humor

b. Upset

1. Patient thinks nothing more can be done

2. Roommate/HCP

12. Patient needs

a. Hope/Support

b. Long distance call

c. Knowledge on i.e. blood sugar

d. Assist with food/drink/activities of daily living/safety

e. Short term memory exercises

f. Music/DVDs/books

g. Exercise

h. Spiritual

13. Test/discharge

a. Delay

b. Sooner than expected

14. HCP interruption

a. HCP wait his/her turn

b. HCP interrupts another HCP - patient interaction

15. Disability

a. Patient already has disability coverage

b. Patient's disability status pending

16. Complications during stay

a. Apology from HCP

b. No apology from HCP

17. Quiet Time 
APPENDIX H

IRB Approval

TheresaGalakatos, MSN-RNandMSW

TileExperienceofKnowledgeAcquisitionforHeartFailurePatientsinan AcuteCareSetting

\section{Assurances}

Principallnvestigator $(\mathrm{PI}) A s P I$, lassurethat:

- Iamultimatelyresponsiblefortheconductofthestudy.

- Iagreetocomplywithallapplicable<UNIVERSITY>policiesandprocedures, andapplicablefederal,state andlocallaws.

- Theapplicationisconsistent withproposal(s) submittedtoexternalfundingagencies.

- Theresearchwillonlybeperformedbyqualifiedpersonnel.

- Allpersonsassistingwiththeresearchareadequatelyinformedabouttheprotocolandtheirresearch-related dutiesandfunctions.

- Iwillnotimplementany changesintheapprovedIRBapplication,studyprotocol,orinformedconsentprocess withoutpriorIRBapproval(exceptinanemergency, ifnecessarytosafeguardthewell-beingofahuman participant).

- Ifunavailabletoconductthisresearchpersonally, aswhenonsabbaticalleave, I willarrangeforanother investigatortoassumedirectresponsibilityforthestudy. Eitherthispersonisnamedasanotherinvestigatorin thisapplication,or I willnotifythelRBofsucharrangements.

- I willobtainContinuingReviewapprovalpriorto12:01amonthedatetheapprovalforthestudyexpires.I understandifIfailtoapplyforcontinuingreview, approvalforthestudywillautomaticallyexpire, andallstudy activitymustcease until IRBapprovalisgranted.

- Theresearchteamwillonlycollectinformationessentialtothestudy.Tothegreatest extentpossible,accessto theinformationwillbelimitedwithintheresearchteam. Ifprotectedhealthinformationisusedorcreated, itwill notbere-usedordisclosedtoanyotherpersonorentity, exceptasrequiredbylaw, researchoversight, orthose usesoutlinedintheapplication.

- Ifmembersoftheresearchteamaccessprotectedhealthinformationinordertoseekconsent authorizationfor research,suchaccessisnecessaryfortheresearch,issolelyforthatpurpose, andtheinformationwillnotbe removedfromthecoveredcomponent.

- Neitherlnoranymemberoftheresearchteam hasafinancialinterest, asdefinedbythe<UNIVERSITY'S>conflictofinterestpolicies, wherebythevalueofthein teresttomeoranymemberoftheresearchteamcouldbe influencedbytheoutcomeofthestudy.AnyrealorpotentialconflictsofinterestthatexistforthePlorany memberoftheresearchteamthatmightaffecttherelationship withtheresearchparticipantortheoutcomeofthe researchwillbedisclosedinaccordancewithinstitutionalpoliciesandappropriatelymanaged,reduced, or eliminated,incooperationwith<UNIVERSITY'S>DisclosureReviewCommittee.

- Ifurtherassurethattheproposedresearchisnotcurrentlybeingconductedandwillnotbeginuntil IRBapproval hasbeenobtained.

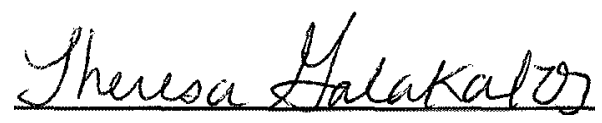

SignatureofPrincipallnvestigator Date

$$
4-2-14
$$

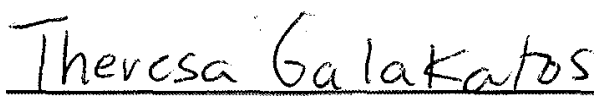

PrintedNameofthePrincipallnvestigator

Dean/DepartmentChairMysignatureassuresthat:

- Theinvestigatorisqualifiedtoconducttheresearchasdescribedinthisapplication.

- Theinvestigatorhasadequateresources, budget,facilities, andnumbersofqualifiedstafftoconducttheresearch asdescribedinthisapplication.

- Ascientificreviewoftheresearchwasconductedandanyrequiredchangesresultingfromtherevieware includedinthesubmittedapplication.

- Theresearchusesproceduresconsistentwithsoundresearchdesign.

- Theresearchdesignissoundenoughtoyieldtheexpectedknowledge. 
- Theinvestigatorhasavailabletimetooverseeandconductthisproject.

- Iftheinvestigatorleaves $<U N I V E R S I T Y>$ withoutnotifyingtheIRB, I willcompletethenecessaryformsto eitherclosethestudyorcontinuethestudyunderthedirectionofadifferentinvestigator.

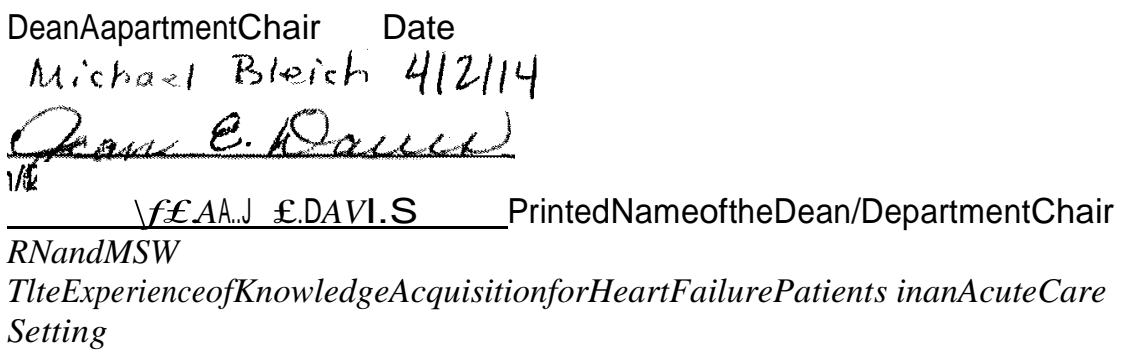

TlteresaGa/akatos, MSN-

FacultySponsor(IfPIisastudent)Thefacultysponsormustbeamemberofthe $<$ UNIVSERSITY'S $>$ facultyand isconsideredtheresponsiblepartyforthescientific,legalandethicalperformanceoftheproject.

Asthefacultysponsoronthisresearchapplication, lassurethat:

- Iwillmeetwiththestudentinvestigatoronaregularbasisandmonitorstudyprogress.

- Thestudentisknowledgeableabouttheregulationsandpoliciesgoverningresearchwithhumansubjectsand hassufficient trainingandexperiencetoconductthisparticularstudyinaccordwiththeapproved protocol.

- If Iwillbeunavailabletosupervisethisresearchpersonally, aswhenonsabbaticalleave, I willarrangeforan alternateFacultySponsortoassumedirectresponsibilityinmyabsenceand I willadvisetheIRBinadvanceof sucharrangements.

- Ifthestudentleaves<UNIVERSITY> withoutnotifyingtheIRB, I willcompleteanyrequiresforms necessarytoeitherclosethestudyorcontinuethestudysolelyundermydirectionornameanotherstudent investigator.

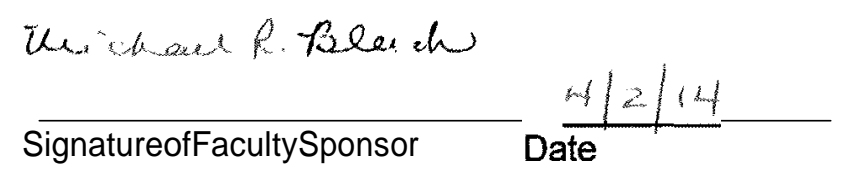

PrintedNameoftheFacultySponsor

\section{ACKNOWLEDGEMENTS}

I would like to express my deepest appreciation to my doctoral chair Dr. Michael Bleich. Hisguidance, caring, and patience provided the perfect atmosphere for completing this doctoral journey. I will always remember his tenacious sense of genius and boundless energythat continually and convincingly strengthened meand lifted up my research to the highest standards. I would like to thank all my committee members, Professor Drinkard, Professor Ward, Professor Davis, and Professor Vaughn, for their unselfish investment in my personal growth and faithful commitment to and support of my research. An intellectual group, each withquick witted comments thatencouraged and inspired me along the way.

This acknowledgement would not be complete without mention of Debbie Mettlach, Margaret O'Connor, Trenise Nelson, Martha Stewart, and Karen Porter who were guardian angels knowing just how to make everything work perfectly.I also credit my mother, Anna Harris, who watched over me through this doctoral journey and for whose love and support was a constant force at every turning point. I would like to thank my father, Dr. Andrew Galakatos, whose experience in health care and educationwas a beacon of lightin this journey. Thank you to my sister, brothers, sisters in law, all of my nieces and nephews, aunts, uncles, cousins, and special prayers of thanks tothose who are no longer with me today, especiallymy stepfather Whitney Harris and my grandmothers Cleopatra Galakatos and Augusta Barwick.Finally, thank you to the administrators, health care providers, and patients at the hospital who supported this research. Their openness and trust is to be honored and revered

\section{REFERENCES}

[1] Abramson, J., Williams, S., \& Krumholz, H. (2001). Moderate alcohol consumption and

[2] risk of heart failure among older persons. JAMA, 285(15), 1971-77.

[3] Albert, N. (2008). Improving medication adherence in chronic cardiovascular disease. Critical Care Nurse, 5, 55-65.

[4] Albert, N., Collier, S., Sumodi, V., Wilkinson, S., Hammel, J., Vopat, L., Willis, C., \&Bittel, B. (2002). Nurses' knowledge of heart failure education principles. Heart \& Lung.31,102-12. 
[5] Albert, N., Trochelman, K., Meyer, K., \&Nutter, B. (2009). Characteristics associated with racial disparities in illness beliefs of patients with heart failure. Behavioral Medicine, 35, 112-125.

[6] Alehagen, U., Rahmqvist, M., Paulsson, T., \& Levin, L. (2008). Quality adjusted life year weights among elderly patients with heart failure. European Journal of Heart Failure, 10, 1033-39.

[7] Alexiades, M. (1996). Selected guidelines for ethnobotanical research: A field manual. New York: New York Botanical Gardens.

[8] American Heart Association. (2012). Your heart failure healthcare team. Retrieved from http://www.heart.org/HEARTORG/Conditions/HeartFailure/PreventionTreatmentofHeartFailure/Your-Heart-FailureHealthcare-Team_UCM_306361_Article.jsp

[9] American Heart Association. (2011). Get with the guidelines: Heart failure. Enhanced heart failure patient education prior to hospital discharge. Retrieved from http://www.heart.org/idc/groups/heartpublic/@wcm/@private/@hcm/@gwtg/documents/downloadable/ucm_428949.pdf

[10] American Heart Association. (2011). Target: HF. Taking the failure out of heart failure. Retrieved from http://www.heart.org/idc/groups/heartpublic/@wcm/@private/@hcm/@gwtg/documents/downloadable/ucm_428949.pdf

[11] American Heart Association. (1994). The criteria committee of the New York Heart Association. Nomenclature and criteria for diagnosis of diseases of the heart and great vessels. Boston: Little, Brown, \& Company, 253-6.

[12] American Hospital Association, TrendWatch. (September, 2011). Examining the drivers

[13] of readmission and reducing unnecessary readmission for better patient care.

[14] Retrieved from

[15] http://webcache.googleusercontent.com/search?q=cache:Ydgh1DMa87wJ:www.aha.org/research/reports/tw/11septw-readmissions.pdf $+\& \mathrm{~cd}=3 \& \mathrm{hl}=\mathrm{en} \& \mathrm{ct}=\mathrm{clnk} \& \mathrm{gl}=\mathrm{us}$

[16] Appleton, J. \& King, L. (1997). Constructivism: A naturalistic methodology for nursing inquiry. Advances in Nursing Science, 20, 13-22.

[17] Anderson, L. (2012). Critical reflection on practice development: Mind your language: Lessons for practice development. International Practice Development Journal, 2, 1-5.

[18] Baker, D., DeWalt, D., Schillinger, D., Hawk, V., Ruo, B., Bibbins-Domingo, K., . .Pignone, M. (2011). "Teach to goal": Theory and design principles of anintervention to improve heart failure self management skills of patients with low health literacy. Journal ofHealth Communication, 16, 73-88.

[19] Baker, D., Parker, R., Williams, M., \& Clark, W. (1998). Health literacy and the risk of hospital admission. Journal of General Internal Medicine, 13, 791-8.

[20] Baker, D., Wolf, M., Feinglass, J., Thompson, J., Gazmararian, J., \& Huang, J. (2007). Health literacy and mortality among elderly persons. Archives of Internal Medicine, 167,1503-9.

[21] Bandura, A. (1986). Social foundations of thought and actions. Englewood Cliffs, NJ: Prentice Hall.

[22] Barrett, S., Puryear, J., \&Westpheling, K. (2008). Health literacy practices in primary care settings: Examples from the field. The Commonwealth Foundation. Retrieved from www.commonwealthfund.org or at www.clinicans.org.

[23] Beckelman, D., Nowels, C., Retrum, J., Allen, L., Shakar, S., Hutt, E., . . Kutner, J. (2011). Giving voice to patients' and family caregivers; needs in chronic heart failure: Implications for palliative care programs. Journal of Palliative Medicine, 14, 1317-24.

[24] Beilby, J. (2004). Definition of metabolic syndrome: Report of the National Heart, Lung, And Blood Institute/American Heart Association conference on scientific issues related to definition. The Clinical Biochemist Review, 25(3), 195-8.

[25] Benderly, M., Haim, M., Boyko, V., Goldbourt, U. (2013). Socioeconomic status indicators and incidence of heart failure among men and women with coronary heart disease. Journal of Cardiac Failure, 19, 117-24.

[26] Bernard, H., Pelto, P., Werner, O., Boster, J., Romney, A., Johnson, A., . . Kasakoff, A. (1986).The construction of primary data in cultural anthropology. Current Anthropology, 27(4),382-96.

[27] Blustein, J., Valentine, M., Mead, H., \& Regenstein, M. (2008). Race/ethnicity and patient confidence to self manage cardiovascular disease. Medical Care, 46, 924-9.

[28] Boren, S., Wakefield, B., Gunlock, T., \& Wakefield, D. (2009). Heart failure self-management education: a systemic review of the evidence. International Journal of Evidence Based Healthcare, 7(3), 159-68.

[29] Boyde, M., Song, S., Peters, P., Turner, C., Thompson, D., \& Stewart, S. (2013). Pilottesting of a self-care education intervention for patients with heart failure. EuropeanJournal of Cardiovascular Nursing, 12(1), 39-46.

[30] Campinha-Bacote, J. (2007). The process of cultural competence in the delivery of healthcare services: The journey continues $\left(5^{\text {th }}\right.$ ed.). Cincinnati, OH: Transcultural C.A.R.E. Associates.

[31] Chapa, D., Akintade, B., Son, H., Woltz P., Hunt, D., Friedmann, E., . . Thomas, S.

[32] (2014). Pathophysiological relationship between heart failure and depression and

[33] anxiety. CriticalCare Nurse, 34(2), 14-25.

[34] Chaudhry, S., Herrin, J., Phillips, C., Butler, J., Mukerjhee, S., Murillo, J., . . . Krumholz, H. (2011). Racial disparities in health literacy and access to care among patients with heart failure. Journal of Cardiac Failure, 17, 122-7.

[35] Chen, A., Yehle, K., Plake, K., Murawski, M., \& Mason, H. (2011). Health literacy and self-care of patients with heart failure. Journal of Cardiovascular Nursing, 26(6), 446-51. 
[36] Chick, K.,Negley, K.,Sievers, B., \&Tammel, K. (2012). Enhancing patient educationthrough clinical nurse specialist collaboration. Clinical Nurse Specialist: The Journal for Advanced Nursing Practice, 26, 317-22.

[37] Clark, N., Gong, M., Schork, A., Evans, D., Roloff, D., Hurwitz, M., . . Mellins, R. (1998). Impact of Education for physicians on patient outcomes.Pediatrics, 101, 831-6.

[38] Collins, S., Pang, P., Fonarow, G., Yancy, C., Bonow, R., \&Gheoghiade, M. (2013). Is hospital admission for heart failure really necessary? The role of the emergency department and observation unit in preventing hospitalization and rehospitalization. Journal of the American College of Cardiology, 61, 121-6.

[39] Creswell, J. (2009). Research design: Qualitative, quantitative, and mixed methods approaches (3 ${ }^{\text {rd }}$ ed.). Thousand Oaks, CA: Sage Publications, Inc.

[40] Creswell, J. \& Clark, V. (2011). Designing and conducting mixed methods research. Thousand Oaks, CA: Sage Publications.

[41] Department of Health and Human Services, Missouri Information for Community Assessment (DHHS/MICA). (2012). Retrieved fromhttp://health.mo.gov/data/mica/mica/chronic_death.php

[42] Department of Health and Human Services, Missouri Information for Community Assessment (DHHS/MICA). (2013). Retrieved from http://health.mo.gov/data/mica/mica/preventable.php

[43] Desai, A. (2012). The three-phase terrain of heart failure readmissions. Circulation: HeartFailure, 5, 389-400.

[44] Dewar, B. (2013). Cultivating compassionate care. Nursing Standard, 27, 48-55.

[45] Driscoll, A., Davidson, P., Clark, R., Huang, N., \& Aho, Z. (2009). Tailoring consumer resources to enhance selfcare in chronic heart failure. Australian Critical Care, 22, 133-40.

[46] Duhamel, F., Dupuis, F., Reidy, M., \&Nadon, N. (2007). A qualitative evaluation of a family nursing intervention. Clinical Nurse Specialist, 21, 43-9.

[47] El Badawy, A. \& El Hefnawy, K. (2013). Randomized controlled trial of comprehensive nursing intervention on readmission, mortality and quality of life among Egyptian heart failure patients: A 12 month follow up study. Journal of Nursing Education \& Practice, 3, 14-30.

[48] Elwyn, G., Edwards, A., Wensing, M., Hood, K., Atwell, C., \&Grol, R. (2003). Shared decision making: Developing the OPTION scale for measuring patient involvement. Quality Safety Health Care, 12, 93-9.

[49] Erlandson, D., Harris, E., Skipper, E., \& Allen, S. (1993). Doing naturalistic inquiry: Aguide to methods. Newbury Park, CA: Sage Publications, Inc.

[50] Evangelista, L. \&Shinnick, M. (2008). What do we know about adherence and self care? Journal of Cardiovascular Nursing, 23, 1-12.

[51] Fallis, B., Dhalla, I., Klemensberg, J., \& Bell, C. (2013). Primary medication non-adherence after discharge from a general internal medicine service. PLoS One, 8 , e61735.

[52] Fleming, M. \& Haney, T. (2013). Improving patient outcomes with better care transitions: The role for home health. Cleveland Clinic Journal of Medicine, 80(1),eS2-6.

[53] Fonarow, G., Albert, N., Curtis, A., Stough, W., Gheorghiade, M., Heywood, J., . . Yancy, C. (2010). Improving evidence based care for heart failure in outpatient cardiology practices: Primary results of the registry to improve the use of evidence based heart failure therapies in the outpatient setting (IMPROVE HF). Circulation, 122, 585-96.

[54] Fowler, S. (2012). Improving community health nurses' knowledge of heart failureeducation principles. Home Healthcare Nurse, 30, 91-101.

[55] Fredericks, S.,Beanlands, H., Spalding, K., \&Da Silva, M. (2010). Effects of thecharacteristics of teaching on the outcomes of heart failure education interventions: Asystematic review. European Journal of Cardiovascular Nursing, 9, 30-7.

[56] Gallacher, K., May, C., Montori, V., \&Mair, F. (2011). Understanding the patients' treatment burden in chronic heart failure using normalization process theory. Annals of Family Medicine, 9, 235-43.

[57] Gesell, S., Clark, P.,\&Williams, A. (2004). Inpatient heart failure treatment from thepatient's perspective. Quality Management in Health Care, 13, 154-65.

[58] Glanz, K., Rimer, B., \&Viswanath, K. (2008). Health behavior and health education: Theory, research, and practice. San Francisco, CA: Jossey Bass.

[59] Go, A., Mozaffarian, D., Roger, V., Benjamin, E., Berry, J., Borden W., . . Turner, M. (2013). Heart disease and stroke statistics-2013 update: A report from the American Heart Association. Circulation,127, e6-e245.

[60] Goodlin, S. (2009). Palliative care in congestive heart failure. Journal of the AmericanCollege of Cardiology, 54, 386-396.

[61] Grady, K., Dracup, K., Kennedy, G., Moser, D., Piano, M., Stevenson, L., \& Young, J. (2000). Team management of patients with heart failure: A statement for healthcare professionals from the Cardiovascular Nursing Council of the American Heart Association. Circulation, 102, 2443-2456.

[62] Guba, E. \& Lincoln, Y. (1994). Competing paradigms in qualitative research. In Denzin, N. \& Lincoln, Y (Eds.), Handbook of qualitative research (pp. 105-17). Thousand Oaks, CA: Sage.

[63] Guskey, T. (1985). Implementing mastery learning. Belmont, CA: Wadsworth.

[64] Haase, J. \& Myers, S. (1988). Reconciling paradigm assumptions of qualitative and quantitative research. Western Journal of Nursing Research, 10, 128-137.

[65] Hain, D., Tappen, R., Diaz, S., \&Ouslander, J. (2012). Cognitive function and medication self management errors in older adults discharged from a community hospital. In Journalof Cardiovascular Nursing, 27, 369. 
[66] Hall, M., Levant, S., and DeFrances, C. (2012). Hospitalization for congestive heart failure: United States, 20002010. National Center for Health Statistics, 108, 1-8. Retrieved from http://www.cdc.gov/nchs/data/databriefs/db108.pdf

[67] Hallerbach, M., Francoeur, A.,Pomerantz, S., Oliner, C., Morris, D., Eiger, G. . . Goldfinger, M. (2008).Patterns and predictors of early hospital readmission in patients with congestive heart failure. American Journal of Medical Quality, 23, 18-23.

[68] Hawkins, N., Jhund, P., McMurray, J., \&Capewell, S. (2012). Heart failure and socioeconomic status: Accumulating evidence of inequality. European Journal of HeartFailure, 14, 138-46.

[69] Heart failure. (2012). In NIH.gov. Retrieved from http://www.nhlbi.nih.gov/health/health-topics/topics/hf/

[70] Heidenrich, P., Trogdon, J., Khavjou, O., Butler, J., Dracup, K., Ezekowitz, M., . . Woo, Y. (2011). Forecasting the future of cardiovascular disease in the United States: A policy statement from the American Heart Associaton. Circulation, 123(8), 933-44.

[71] Helleso, R., Eines, J., Sorensen, L., \&Fagermoen, M. (2009). Severity of illness: Implications for information management by patients. Studies in Health Technology and Informatics, 146, 373-7.

[72] Henry Kaiser Family Foundation. (2012). The hospital adjusted expenses per inpatient days. Retrieved from http://kff.org/other/state-indicator/expenses-per-inpatient-day/\#table

[73] Hines, P., Yu, K., \& Randall, M. (2010). Preventing heart failure readmissions: Is your organization prepared? Nursing Economics, 28, 74-86.

[74] How will the uninsured in Missouri fare under the Affordable Care Act? (2014). The Henry J. Kaiser Foundation. Retrieved from www.kff.org/health-reform/fact-sheet/state-profiles-uninsured-under-aca-missouri/

[75] Hunt, S. (2005). American College of Cardiology; American Heart Association Task Force on Practice Guidelines (Writing Committee to Update the 2001 Guidelines for the Evaluation and Management of Heart Failure). Journal of the American College of Cardiology, 46, e1-82.

[76] Institute of Medicine. (2004). Report Brief. Health literacy: A prescription to end confusion.Retrieved from http://www.iom.edu/ /media/Files/Report\%20Files/2004/Health-Literacy-A-Prescription-to-EndConfusion/healthliteracyfinal.pdf

[77] Institute of Medicine. (2010). The future of nursing: Leading change, advancing health. Retrieved from http://books.nap.edu/openbook.php?record_id=12956\&page=R1

[78] Institute of Medicine (2011). The future of nursing. Retrieved from http://www.iom.edu/Reports/2010/The-Future-ofNursing-Leading-Change-Advancing-Health/Infographic.aspx

[79] Ishikawa, H., Hashimoto, H., \&Kiuchi, T. (2013). The evolving concept of patient centeredness in patient-physician communication research. Social Science \& Medicine,96, 147-153.

[80] Jencks, S., Williams, M., \& Coleman, E. (2009). Rehospitalization among patients inMedicare fee for service program. New England Journal of Medicine, 360, 1418-28.

[81] Kalogirou, F., Lambrinou, E., Middleton, Nicos, \&Sourtzi, P. (2012). Cypriot nurses'knowledge of heart failure selfmanagement principles.European Journal of Cardiovascular Nursing, 12, 159-66.

[82] Kayaniyil, S.,Ardern, C., Wistanley, J., Parsons, C., Brister, S., Oh, P., . . G Grace, S. (2009).Degree and correlates of cardiac knowledge and awareness among cardiac inpatients.Patient Education \& Counseling, 75, 99-107.

[83] Keteyian, S. (2011). Exercise training in congestive heart failure: Risks and benefits. Exercise andCardiovascular Diseases, Progress in Cardiovascular Diseases,

[84] 53(6), 419-28.

[85] Kochanek, K., Xu, J., Murphy, S., Miniño, A., \& Kung, H. (2011). Deaths: Final data for 2009. National Vital Statistics Reports, 60, 3.

[86] Koekkoek, D., Baley, B., Brown, A., \&Rustvold, L. (2011). Hospitalists assess the causes of early hospital readmissions. Journal of Hospital Medicine, 6, 383-88.

[87] Koelling, T., Johnson, M., Cody, R., \& Aaronson, K. (2005). Discharge education improves clinical outcomes in patients with chronic heart failure. Circulation, 111, 179-85.

[88] Krumholz, H., Baker, D., Ashton, C., Dunbar, S., Friesinger, G., Havranek, E., . . .Spertus, J. (2000). Evaluating quality of care for patients with heart failure. Circulation, 101, e122-e140.

[89] Krumholz, H., Parent, E., Tu, N., Vaccarino, V., Wang, Y., Radford, M., \&Hennen, J. (1997). Readmission after hospitalization for congestive heart failure among Medicare beneficiaries. JAMA Internal Medicine, 157, 99-104.

[90] Kumagai, A. \&Lypson, M. (2009). Beyond cultural competence: Critical consciousness, social justice, and multicultural education. Academic Medicine, 84, 782-7.

[91] Lee, D., Tu, J., Juurlink, D., Alter, D., Ko, D., Austin, P., . . .Laupacis, A. (2005). Risk treatment mismatch in the pharmacotherapy of heart failure. JAMA, 294, 1240-7.

[92] Leininger, M. \& McFarland, M. (2006). Culture care diversity and universality: A worldwide nursing theory (2 $2^{\text {nd }}$ ed.). Sudbury, MA: Jones and Bartlett Publishers, Inc.

[93] Lincoln, Y. \& Guba, E. (1985). Naturalistic inquiry. Newbury Park, CA: Sage Publications.

[94] Lofland, J. \&Lofland, L. (1995). Analyzing social settings: A guide to qualitativeobservations and analysis. Belmont, CA: Wadsworth Publishing.

[95] Lofvenmark, C., Saboonchi, F., Edner, M., Billing, E., \&Mattisson, A. (2012). Evaluationof an educational programme for family members of patients living with heart failure: A randomized controlled trial. Journal of Clinical Nursing, 22, 115-126. 
[96] Manning, S. (2011). Bridging the gap between hospital and home: A new model of care for reducing readmission rates in chronic heart failure. Journal of Cardiovascular Nursing, 25(5), 368-7.

[97] Marshall, C. \&Rossman, G. (2011). Designing qualitative research (5 ed.). Washington, DC: Sage Publications, Inc.

[98] Matlock, D., Nowles, C., \&Bekelman, D. (2010). Patient perspectives on decision making in heart failure. Journal of Cardiac Failure, 16, 823-6.

[99] Mayo Clinic. (2013). Heart failure: Risk factors. Retrieved on April 7, 2013 from http://www.mayoclinic.com/health/heart-failure/DS00061/DSECTION=risk-factors

[100] McDonald, K., Ledwidge, M., Cahill, J., Quigley, P., Maurer, B., Travers, B., . . Ryan, E.(2002). Heart failure management: Multidisciplinary care has intrinsic benefit above the optimization of medical care.Journal of Cardiac Failure,3, 142-48.

[101] McMurray, J. \& Stewart, S. (2000). Epidemiology, aetiology, and prognosis of heart failure. Heart and Education in Heart, 83, 596-602.

[102] Missouri Department of Health and Senior Services. (2011). 2010-2013 Rural Health Plan.Retrieved from http://health.mo.gov/living/families/ruralhealth

[103] Missouri Department of Health and Senior Services. (2007). The behavioral risk factor surveillance system survey. Retrieved from http://www.cdc.gov/DHDSP/programs/nhdsp_program/mo.htm

[104] Morrow, D., Clark, D., Wanzhu, T., Jinwei, W., Weiner, M., Steinley, D., \& Murray, M. (2006). Correlates of health literacy in patients with chronic heart failure. The Gerontologist, 46(5), 669-76.

[105] Moser, D., Lee, K., Jia-Rong, W., Mudd-Martin, G., Jaarsma, T., Huang, T., . . Riegel,

[106] B. (2014). Identification of symptom clusters among patients with heart failure:

[107] An international observational study. International Journal of Nursing Studies,

[108] 51(10), 366-72.

[109] Moser, D. \& Mann, D. (2002). Improving outcomes in heart failure: It is not unusual beyond usual care. Circulation, 105, 2810-2812.

[110] Murray, M., Young, J., Hoke, S., Tu W., Weiner, M., Morrow, D., . . Brater, D. (2007). Pharmacist intervention to improve medication adherence in heart failure. Annals of InternalMedicine, 146(10),714-25.

[111] Morrow, D., Clark, D., Tu, W., Wu, J., Weiner, M., Steinley, D., \& Murray, M. (2006). Correlates of health literacy in patients with chronic heart failure. The Gerontologist, 46(5), 669-76.

[112] Paradis, V., Cossette, S., Frasure-Smith, N., Heppell, S. \&Guertin, M. (2010). The efficacy of a motivational nursing intervention based on the stages of change on self-care in heart failure patients. Journal of Cardiovascular Nursing, 25, 130-41.

[113] Patton, M. (1999). Enhancing the quality and credibility of qualitative analysis. HSR: Health Services Research, 34, $1189-1208$.

[114] Parker, R., \& Ratzan, S. C. (2010). Health literacy: A second decade of distinction for Americans. Journal of Health Communication, 15, 20-33.

[115] Peterson, P., Shetterly, S., Clarke, C., Bekelman, D., Chan, P., Allen, L., . . Masoudi, F.

[116] (2011). Health literacy and outcomes among patients with heart failure. JAMA,

[117] 305(16), 1695-1701.

[118] Phillips, C., Wright, S., Kern, D., Singa, R., Shepperd, S., \& Rubin, H. (2004). Comprehensive discharge planning with post discharge support for older patients with congestive heart failure: A meta-analysis. JAMA, 291, 1358-67.

[119] Polit, D. \&Hungler, B. (1999). Nursing research: Principles and methods (6 ${ }^{\text {th }}$ ed.). $\quad$ Philadelphia, PA: Lippincott.

[120] Portney, L. \& Watkins, M. (2009). Foundations of clinical research: Applications to practice ( ${ }^{\text {rd }}$ ed.). Upper Saddle River, NJ: Pearson Prentice Hall.

[121] Pugh, L., Tringali, R., Boehmer, J., Blaha, C., Kruger, N., Capauna, T., . . Xie, S. (1999).Partners in care: A model of collaboration. Holistic Nursing Practice 13, 61-5.

[122] Purden, M. (2005). Cultural considerations in interprofessional education and practice. Journal of Interprofessional Care, 1, 224-234.

[123] Radhakrishnan, K., \&Jacelon, C. (2012). Impact of tele health on patient self-management of heart failure: A review of literature. Journal of Cardiovascular Nursing, 27(1), 33-43.

[124] Readability Score. (n.d.). Retrieved from https://readability-score.com

[125] Reduced heart failure length of stay. (n.d.). $\quad$ Retrieved from http://www.juran.com/elifeline/elifefiles/2013/02/Juran_CS_ReduceHeartFailureLengthofStay_2012.pdf

[126] Reigel, B., Carlson, B., Kopp, Z., LePetri, B., Glaser, D., \& Unger, A. (2002). Effect of a standardized nurse case management telephone intervention on resource use in patients with chronic heart failure. Archives of Internal Medicine, 162, 705-12.

[127] Reardon, L. \&Pillutia, P. (2013). Congenital Heart Disease Conference. In Jamil Abulhousn (Chair), Heart failure and pulmonary hypertension. Symposium conducted at the meeting of Ahamson, UCLA Health System.

[128] Richards, L., \& Morse, Janice, M. (2007). Readme first for a user's guide to qualitative methods $\left(2^{\text {nd }}\right.$ ed.). Thousand Oaks, CA: Sage Publications.

[129] Richards, L. \&Nirsem J. (2007). Readme first for a user's guide to qualitative methods ( $2^{\text {nd }}$ ed.). Thousand Oaks, CA: Sage Publications, Inc.

[130] Robinson, S., Moser, D., Pelter, M., Nesbitt, T., Paul, S., \&Dracup, K. (2011). Assessing health literacy in heart failure patients. Journal of Cardiac Failure, 7(11), 887-892. 
[131] Rogers, V., Go, A., Lloyd-Jones, D., Benjamin, E., Berry, J., Borden, W., . . Turner, M. (2012). Heart disease and stroke statistics-2012 update: A report from the American HeartAssociation statistics committee and stroke statistics subcommittee. Circulation, 125, e2-220.

[132] Rogers, V., Go, A., Lloyd-Jones, D., Adams, R., Berry, G., Brown, T., . . Wylie-Rosett, J. (2011). Heartdisease and stroke statistics-2011 update: A report from the American Heart Associationstatistics committee and stroke statistics subcommittee.Circulation, 123(4)18-209.

[133] Rogers, A., Addington-Hall, J., Abery, A., McCoy, A., Bulpitt, C., Coats, A., \& Gibbs, J. (2000).Knowledge and communication difficulties for patients with chronic heart failure: Qualitative study. BMJ, 321(7261),605-7.

[134] Rogers, A., Addington-Hall, J., McCoy, A., Edmonds, P., Abery, A., Coats, J., \& Gibbs, J. (2002). A qualitative study of chronic heart failure patients' understanding of their symptoms and drug therapy. European Journal of Heart Failure, 4(3), 283-287.

[135] Roncalli, J., Perez, L., Pathak, A., Spinazze, L., Mazon, S., Lairez, O. . . Galinier, L. (2009). Improvement of young and elderly patient's knowledge of heart failure after an educational session. Cardiology, 3, 45-52.

[136] Rosland, A., Heisler, M., \&Piette, J. (2012). The impact of family behaviors and communication patterns on chronic illness outcomes: A systematic review. Journal of Behavioral Medicine, 35(2), 221-39.

[137] Schoen, C., Osborn, R., Huynh, P., Doty, M.,Zapert, K., Peugh, J., \& Davis, K. (2005). Takingthe pulse of health care systems: Experiences of patients with health problems in six countries. Health Affairs, W5-509-25.

[138] Schatzman, L. \& Strauss, A. (1973). Field research: Strategies for natural sociology. $\quad$ Englewood Cliffs, NJ: Prentice Hall.

[139] Schrager, J., Wheatley, M., Georgiopoulou, V., Osborne, A., Kalogeropoulos, A., Hung, O., . . Ross, M. (2013). Favorable bed utilization and readmission rates for emergency department observation unit heart failure patients. Academic Emergency Medicine, 20, 554-61.

$\begin{array}{lllllll}\text { [140] SES. (n.d.) } & \text { In } & \text { dictionary.com } & \text { online } & \text { dictionary. } & \text { Retrieved } & \text { from }\end{array}$ http://dictionary.reference.com/browse/socioeconomic+status

[141] Shenton, L. (2004). Strategies for ensuring trustworthiness in qualitative research projects.

[142] Education for Information, 22, 63-75. Retrieved from

[143] http://www.crec.co.uk/docs/Trustworthypaper.pdf

[144] Shi, L.,\& Singh, D. (2011). The nation's health ( $8^{\text {th }}$ ed.). Sudbury, MA: Jones and

[145] Bartlett Learning, LLC.

[146] Skerrett, P. (2012). Studies explore burden of disease and heart disease in the United States. Harvard Health Publications. Retrieved from http://www.health.harvard.edu/blog/studies-explore-global-burden-of-disease-andheart-disease-in-the-united-states-201212145637

[147] Sreih, A. (2004). Spironolactone: The missing drug in the treatment of patients hospitalized with congestive heart failure. Journal of Clinical Outcomes, 11, 568-73.

[148] Smedley, B., Stith, A, \& Nelson, A. (2003). Summary. Unequal treatment. Confronting racial and ethnic disparities in healthcare. Washington, DC: National Academies, 1-28. Socioeconomic status. (2013). Retrieved fromhttp://dictionary.reference.com/browse/socioeconomic+status

[149] Stevenson, W., Stevenson, L., Middlekauff, H., Fonarow, G., Hamilton, M., Woo, M., .. .Tillisch, J. (1995). Improving survival for patients with advanced heart failure: A study of 737 consecutive patients. Journal of the American College of Cardiology, 26(6), 1417-23.

[150] Stewart, S. \& Horowitz, J. (2002). Home based intervention in congestive heart failure: Long term implications on readmission and survival. Circulation, 105, 2861-66.

[151] Street, R., O’Malley, K., Cooper, L., \&Haidet, P. (2008). Understanding concordance in patient - physician relationships: personal and ethnic dimensions of shared identity. Annals of Family Medicine, 6 , 198-205.

[152] Stromberg, A. (2005). The crucial role of patient education in heart failure. The European Journal of Heart Failure, 7, 363-9.

[153] Suh, E. (2004). The model of cultural competence through an evolutionary concept analysis. Journal of Transcultural Nursing, 15, 93-102.

[154] The coverage gap: Uninsured poor adults in states that do not expand Medicaid. (2014). The Henry J. Kaiser Foundation. Retrieved from www.kff.org/health-reform/poor-adults-in-states-that-do-not-expand-medicaid/

[155] Thornhill, K., Lyons, A., Nouwen, A., \& Lip, G. 2008).Experiences of living withcongestive heart failure: A qualitative study. British Journal of Health Psychology 13,155-75.

[156] U.S. Department of Health and Human Services. (2014). Implementation of the Medical Home program. Retrieved from http://mchb.hrsa.gov/programs/medicalhome/

[157] U.S. Department of Health and Human Services. (2013). Retrieved from http://www.healthypeople.gov/2020/topicsobjectives2020/overview.aspx?topicid=21\#two

[158] U.S. Department of Health and Human Service Office of Minority Health (HHS/OMH). (1991). CLAS standards. Retrieved from http://minorityhealth.hhs.gov/omh/browse.aspx?lvl=2\&lvlid=53

[159] Van der Wal, M., Jaarsma, T., Moser, D., Veeger, N., van Gilst, W., \& van Velduisen, D. (2007). Compliance in heart failure patients: The importance of knowledge and beliefs. European Heart Journal, 27, 434-40. 
[160] Washburn, S., Hornberger, C., Klutman, A., \& Skinner, L. (2005). Nurses' knowledge of heart failure education topics as reported in a small Midwestern community hospital. Journal of Cardiovascular Nursing, 20, 215-20.

[161] Whellan, D., Goodlin, S., Dickinson, M., Heidenreich, P., Jaenicke, C., Stough, W., \&Rich, M. (2014). Consensus statement: End of life care in patients with heart failure. Journal of Cardiac Failure, 20(2), 121-34.

[162] Willett, E., Surrells, D., Davis, L., \& Bush, C. (2007). Nurses' knowledge of heart failure self management. Progress in Cardiovascular Nursing, 22, 190-5.

[163] Williams, S., Schmaltz, S., Morton, D., Koss, R., \& Loeb, M. (2005). Quality of care in U.S. hospitals as reflected by standardized measures, 2002-2004. New England Journal of Medicine, 353, 255-64.

[164] Yehle, K. \&Plake, K. (2010). Self-efficacy and educational interventions in heart failure: A review of the literature. Journal of Cardiovascular Nursing, 25, 175-88.

[165] Yilmaz, A., Erdem, A., Kucukdurmaz, Z., Karapinar, H., Gul, I., Sarikaya, S., \&Dizman, R. (2013). Abnormal heart rate recovery in stable heart failure patients. Pacing \&

[166] ClinicalElectrophysiology, 36(5), 591-5.

[167] Zhu, F. \& Levin, N. (2011). Dry weight and measurements methods. In Penido, M.,

[168] Hemodialysis: Different aspects, (pp. 263-84). Retrieved from

[169] http://cdn.intechopen.com/pdfs-wm/23806.pdf 